\title{
Phase diagram of ultrathin ferromagnetic films with perpendicular anisotropy
}

\author{
Ar. Abanov, V. Kalatsky, and V. L. Pokrovsky \\ Department of Physics, Texas A\&M University, College Station, Texas 77843-4242 \\ and Landau Institute for Theoretical Physics, Kosygin str.2, Moscow 117940, Russia \\ W. M. Saslow \\ Department of Physics, Texas A\&M University, College Station, Texas 77843-4242
}

(Received 31 May 1994)

\begin{abstract}
Ultrathin ferromagnetic films with perpendicular spin anisotropy can possess an alternating up-down stripe-domain structure, with widths $L \pm \delta$. Considering the two inequivalent types of stripe domains to form a single unit, this structure may be thought of as a two-dimensional smectic crystal. It is subject to a weak stripe orientation energy. With increasing temperature the domain system changes from a smectic crystal phase to an "Ising nematic" phase, and then to a "tetragonal liquid" phase. We discuss its possible phase diagrams, in $\left(H_{\perp}, H_{\|}, T\right)$ space. This sequence of phases can occur whether or not the system ultimately undergoes a spin reorientation transition to a planar phase.
\end{abstract}

\section{INTRODUCTION}

A consistent physical picture has recently emerged ${ }^{1,2}$ to explain the unusual physical properties of numerous ultrathin ferromagnetic films that show (1) a planar magnetization for temperature $T$ above the spin reorientation transition temperature $T_{R},(2)$ a perpendicular magnetization at low temperatures, and (3) an intermediate temperature regime having a perpendicular magnetization with an up-and-down stripe-domain structure. ${ }^{3-9}$ It is based upon the usual ferromagnetic exchange Hamiltonian, including the magnetostatic dipolar interaction, supplemented by an easy-axis spin anisotropy at the surface that favors spin orientation normal to the plane. ${ }^{10,11}$ The stripe-domain structure serves to minimize the magnetic dipole energy, with the stripe width limited by the energy it costs to form a conventional domain wall, which separates up and down stripes. (Later we will discuss another type of domain wall, which we call a stripe rotation domain wall, separating regions where the stripes are mutually perpendicular.) One of the predictions of the theory, in agreement with experiment, is that the stripe width is strongly dependent upon temperature $T$, growing to fill the entire sample at low temperatures. ${ }^{1,2}$ Another prediction, not yet verified by experiment, is that there is a phase transition from a stripe-domain phase with stripe orientation order and algebraically decaying spatial order to a phase with orientational order and exponentially decaying spatial order, which we call an Ising nematic.

In this work we present a more comprehensive picture of the stripe-domain structure. In addition to further elucidating the properties of the Ising nematic phase, we find that at a higher temperature, but still below $T_{R}$, there is yet another phase based upon the stripe local order, which we call a tetragonal liquid, in which even the stripe orientation order decays exponentially. (Tetrago- nal applies because the substrate provides a stripe orientation anisotropy that favors only two, rather than a continuum, of preferred stripe orientations.) We also present results appropriate to the stripe-domain structure in a nonzero magnetic field $H$. In that case, the alternating up-down stripe-domain structure should possess unequal width $L \pm \delta,{ }^{1,2}$ where $L$ and $\delta$ both depend upon $H$ and $T$. We denote by $u_{s}$ and $v_{s}$ the displacements of the left and right domain walls bordering the $s$ th stripe with equilibrium width $L+\delta$. Considering the two inequivalent types of stripe together, as a layer of thickness $2 L$, this is a two-dimensional (2D) smectic-like crystal at temperature $T=0$. See Fig. 1 .

We now present an overview that emphasizes the simpler case when $H=0$, where only the variable $u$ need be discussed. In practice, because of the sensitivity to $H$, both $u$ and $v$ must be included.

Let the stripe density, in equilibrium, be $n=L^{-1}$. Considering the stripes to be aligned along the local $y$ direction, so that moving along the local $x$ direction takes us from stripe to stripe, changes in the stripe density are then given by $\delta n / n=-\delta L / L$. Going to the continuum form $u(x)$, this corresponds to $\partial_{x} u \approx\left(u_{s+2}-u_{s}\right) / 2 L$ $=\delta L / L=-\delta n / n$. A continuum theory of the dependence on $u$ of the conventional domain wall energy and on the magnetic dipole energy already has been developed in Refs. 1 and 2, involving energy densities associated with compression $\left(\partial_{x} u\right)$ and bending $\left(\partial_{y}^{2} u\right)$. In addition, fourth-order gradient terms in the exchange energy within the conventional domain walls yield a continuum stripe orientation anisotropy energy density that tends to stabilize the conventional domain-wall orientation (within the plane), denoted by $\theta$, where $\tan \theta=\partial_{y} u$, also discussed in Refs. 1 and 2. From the continuum energy and the associated elastic constants, the thermal fluctuations and their effect on the system can be determined. Unless indicated to the contrary, in what follows 


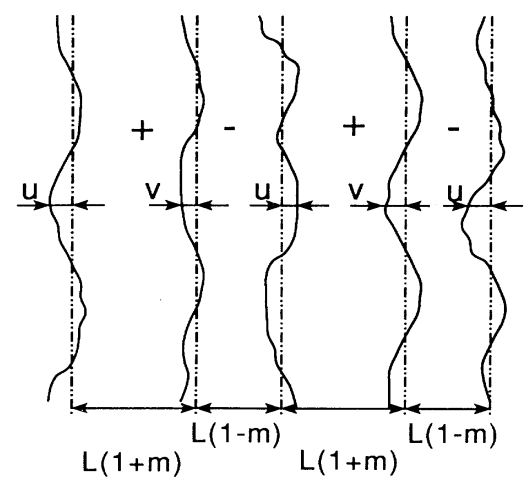

FIG. 1. The geometry of the stripe-domain structure.

the word orientation will refer to orientation of the stripe domains, to be distinguished from the spin orientation (since the spins within each stripe are normal to the plane). There are a number of phases with distinct types of order.

(1) At low temperatures, the stripe domains form what may be described as an oriented smecticlike crystal, where the stripe orientation anisotropy favors only two, mutually perpendicular, directions. Long-wavelength fluctuations cause the spatial order to fall off algebraically with distance. This phase also supports topological excitations: bound dislocation pairs with equal and opposite Burgers vectors $\pm 2 L$. A dislocation in this system corresponds to the partial insertion into the structure of a smectic unit of width $2 L$. See Fig. 2. (Without the stripe orientation energy, the long-wavelength fluctuations would cause the spatial order to decay exponentially, and individual dislocations would have a finite energy.)

(2) Thermally excited bound dislocation pairs become more common as the temperature is increased, until a Berezinskii-Kosterlitz-Thouless (BKT) transition occurs at a temperature $T_{P}$, at which unbound dislocations proliferate and thus the algebraic positional order $(P)$ disappears. ${ }^{12,13}$ Despite this loss of algebraic positional order,

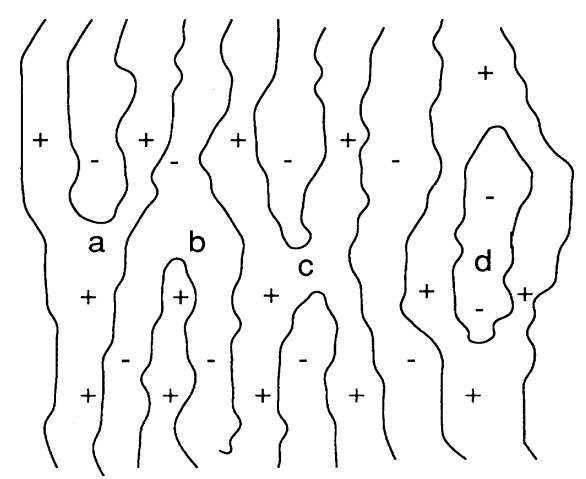

FIG. 2. Dislocations in the stripe-domain structure. (a) dislocation inserted from above; (b) dislocation inserted from below; (c) "strait," or "passage," due to two dislocations, one inserted from above and one from below; (d) "island," due to two dislocations inserted in the center, one ending above and one ending below the center. orientational order persists. Since the stripe orientation energy continues to favor only two, mutually perpendicular, stripe orientations, this may be described as an Ising nematic structure.

(3) The Ising nematic phase is stable over a finite range of temperatures from $T_{P}$ to a temperature $T_{O}$ at which orientational $(O)$ melting occurs. The stripe orientation energy continues to favor only two, mutually perpendicular, stripe orientations, thus making the transition at $T_{0}$ have Ising-like symmetry. Near $T_{O}$, there is a proliferation what we have called a stripe rotation domain wall, within which the stripes rotate from one of the two preferred orientations to the other. Above $T_{O}$, this structure may be described as a disordered tetragonal liquid structure, with unbroken tetragonal symmetry.

An outline of the paper is as follows. Section II presents the microscopic Hamiltonian on which this work is based. Section III contains a detailed discussion of the sequence of phases for $H=0$. Section IV presents a detailed discussion of the properties of the Ising nematic and tetragonal liquid phase, including a very general treatment of the texture associated with stripe rotation domain walls. Section V discusses the KosterlitzThouless transition $T_{P}$ in a field, showing that, in meanfield theory, $T_{P}$ is independent of field. Section VI discusses the phase diagram in $\left(H_{1}, H_{\|}, T\right)$ space. Section VII presents a summary and our conclusions. In Appendix A we compute certain stripe elastic constants for zero field. In Appendix B we compute these same elastic constants for nonzero field. In Appendix $C$ we determine the leading term in the thermal renormalization of the elastic constant $\kappa$.

\section{MICROSCOPIC HAMILTONIAN}

We now present the microscopic Hamiltonian and its continuum limit, which we employ in order to obtain the elastic constants. In going to the continuum limit, we shall consider the in-plane geometry to be that of a square with lattice constant and nearest-neighbor distance $a$. However, we shall also give more general results in terms of the number of spins per unit area $\sigma$ and nearest-neighbor distance $a$.

The microscopic spin anisotropy energy, assumed to be associated only with one surface plane, is given by

$$
\mathscr{H}_{\text {an }}=-D \sum_{i} S_{i z}^{2} \rightarrow-\lambda \int n_{z}^{2} d^{2} x
$$

where we have taken $\mathrm{S}_{\mathrm{i}} \rightarrow S_{\mathrm{n}}$, and $\lambda(T)-D(T)\langle S\rangle^{2} / a^{2}$ for a square lattice; for other lattices $\lambda(T)=D(T)\langle S\rangle^{2} \sigma$.

The total microscopic exchange energy takes the form

$$
\mathscr{H}_{\mathrm{ex}}^{\mathrm{tot}}=-J \sum_{i j} \mathbf{S}_{\mathrm{i}} \cdot \mathbf{S}_{\mathrm{j}} \cdot
$$

In the continuum limit it can be transformed into a constant term (from uniform alignment) plus

$$
\mathscr{H}_{\text {ex }}=\frac{\Gamma(T)}{2} \int\left(\partial_{\alpha} \mathbf{n}\right) \cdot\left(\partial_{\alpha} \mathbf{n}\right) d^{2} x,
$$

where $\Gamma(T)=2 J S^{2}$ for a square lattice; for other lattices, $\Gamma(T)=z J S^{2} / 2$, where $z$ is the number of nearest neigh- 
bors. This energy is the same for each of the $N$ planes.

The magnetic dipolar energy $\mathscr{H}_{\text {dip }}$ is represented by a sum with $\mathbf{m}_{i}=g \mu_{B} \mathrm{~S}_{\mathrm{i}}$, it becomes

$$
\begin{aligned}
\mathscr{H}_{\mathrm{dip}} & =\frac{1}{2} \sum_{i j} \frac{\left(\mathbf{m}_{\mathrm{i}} \cdot \mathbf{m}_{\mathbf{j}}\right)-\mathbf{3}\left(\mathbf{m}_{\mathrm{i}} \cdot v\right)\left(\mathbf{m}_{\mathrm{j}} \cdot v\right)}{\mathbf{x}_{\mathrm{ij}}^{3}} \\
& =\frac{\left(g \mu_{B}\right)^{2}}{2} \sum_{i j} \frac{\left(\mathbf{S}_{\mathrm{i}} \cdot \mathbf{S}_{\mathrm{j}}\right)-\mathbf{3}\left(\mathbf{S}_{\mathrm{i}} \cdot v\right)\left(\mathbf{S}_{\mathrm{j}} \cdot v\right)}{\mathbf{x}_{\mathrm{ij}}^{3}},
\end{aligned}
$$

where $v$ is a unit vector pointing from $\mathbf{x}_{\mathbf{i}}$ to $\mathbf{x}_{\mathbf{j}}$.

We now separate the magnetic dipole energy into a short-range and a long-range part. The short-range part has the form of a single-ion spin anisotropy, and favors in-plane spin orientation. Including the short-range part of the magnetic dipole energy, the total effective spin anisotropy can be written as

$$
\lambda_{\mathrm{eff}}=\lambda-(N \Omega / a) c_{d},
$$

where $c_{d}$ is a dimensionless number on the order of unity for the $N=1$ square lattice, and varying as $\ln N$ for sufficiently large $N$. For stability of the stripe-domain structure, we assume that $\lambda_{\text {eff }}>0$. In addition, for the spin reorientation transition to occur, the temperature dependence of $\lambda_{\text {eff }}$ must be such that it is positive above $T_{R}$ and negative below. ${ }^{14-16}$ The present considerations, however, apply even for systems that do not possess a spin reorientation transition, such as can occur for the thinnest of films.

The long-range part of the magnetic dipole energy can be transformed from summation over sites $i$ and $j$ in three-space to a form in which one integrates over the two-component vectors $\mathbf{x}$ and $\mathbf{x}^{\prime}$ and one sums over the individual layers $I$ and $J$, so $\sum_{i} \mathbf{S}_{\mathrm{i}} \rightarrow \sum_{I} \int\left(d^{2} x / a^{2}\right) S \mathbf{n}$

$$
\begin{aligned}
\mathscr{H}_{\mathrm{dip}} & =\frac{\left(g \mu_{B}\right)^{2}}{2} \sum_{i j} \frac{\left(\mathbf{S}_{\mathrm{i}} \cdot \mathbf{S}_{\mathbf{j}}\right)-\mathbf{3}\left(\mathbf{S}_{\mathrm{i}} \cdot v\right)\left(\mathbf{S}_{\mathrm{j}} \cdot v\right)}{\mathbf{x}_{\mathrm{ij}}^{3}} \\
& \rightarrow \frac{\Omega}{4 \pi} \sum_{I J} \int \frac{\left(\mathbf{n} \cdot \mathbf{n}^{\prime}\right)-3(\mathbf{n} \cdot v)\left(\mathbf{n}^{\prime} \cdot v\right)}{\left|\mathbf{x}-\mathbf{x}^{\prime}\right|^{3}} d^{2} x d^{2} x^{\prime},
\end{aligned}
$$

where $\Omega=2 \pi\left(g \mu_{B} S a^{-2}\right)^{2}$ for the square lattice; for other lattices $\Omega=2 \pi\left(g \mu_{B} S \sigma\right)^{2}$. Here $g$ is the gyromagnetic ratio and $\mu_{B}$ is the Bohr magneton. In the integral, the short-range divergent part is considered to be subtracted; that is, $\left|\mathbf{x}-\mathbf{x}^{\prime}\right|^{-3}$ is considered to be $\left|\mathbf{x}-\mathbf{x}^{\prime}\right|^{-3}-\delta^{(2)}\left(\mathbf{x}-\mathbf{x}^{\prime}\right) \int \mathbf{d}^{2} \mathbf{y}|\mathbf{x}-\mathbf{y}|^{-3}$. The second term, when expressed in Fourier space, is independent of wave vector, and its zero-wave-vector part precisely cancels out the zero-wave-vector part of the first term. The remaining Fourier component of the long-range dipolar interaction goes to zero as $|\mathbf{q}|$, where $\mathbf{q}$ is the wave vector. Nevertheless, it is the dominant interaction at large distances $L>L_{d}$, where

$$
L_{d}=4 \pi \Gamma / \Omega \text {. }
$$

For long-wavelength variations, each term in the sum over $I$ and $J$ is the same, giving a factor of $N^{2}$.

In the continuum limit, one can show that a spin in one plane will have zero interaction with another plane of totally aligned spins. As a consequence, in Eq. (6) we neglect the interaction between spins in different planes. Further, because we shall be interested in the stripedomain phase with spins perpendicular to the film, we may neglect the term $3(v \cdot \mathbf{n})\left(v^{\prime} \cdot \mathbf{n}^{\prime}\right)$ in Eq. (6). Now, by Gauss's law, in the first term one can convert the integrals over the area element $d^{2} x \rightarrow d \mathbf{A}$ to integrals over the surrounding contour element $d l^{2}$. In terms of the displacement $u$, on summing over the interactions of (even) up domains and (odd) down domains, and neglecting any contribution from spins within the conventional domain walls, the magnetostatic energy is given $b y^{2}$

$$
\mathcal{H}_{\text {dip }}=-\sum_{m, n}(-1)^{m-n} \iint V\left(R_{m n}\right) f\left(\partial_{y} u_{m}, \partial_{y}^{\prime} u_{n}\right) d y d y^{\prime} \text {, }
$$

where

$$
\begin{aligned}
& \begin{aligned}
& f\left(\partial_{y} u_{m}, \partial_{y}^{\prime} u_{n}\right) \equiv \cos \left(\partial_{y} u_{m}-\partial_{y}^{\prime} u_{n}\right) \\
& \times \sqrt{1+\left(\partial_{y} u_{m}\right)^{2}} \sqrt{1+\left(\partial_{y}^{\prime} u_{n}\right)^{2}} \\
& \approx\left(1+\partial_{y} u_{m} \partial_{y}^{\prime} u_{n}\right), \\
& V(R)=\frac{\Omega}{\pi \sqrt{R^{2}+l^{2}}},
\end{aligned}
\end{aligned}
$$

and

$$
\begin{aligned}
R_{m n}^{2}= & {\left[(m-n) L+u_{m}(y)-u_{n}\left(y^{\prime}\right)\right]^{2}+\left(y-y^{\prime}\right)^{2} } \\
= & r_{m n}^{2}+2 L(m-n)\left[u_{m}(y)-u_{n}\left(y^{\prime}\right)\right] \\
& +\left[u_{m}(y)-u_{n}\left(y^{\prime}\right)\right]^{2}
\end{aligned}
$$

with

$$
l=\sqrt{\Gamma / 2 \lambda_{\mathrm{eff}}},
$$

being a cutoff to account for the finite width of the conventional domain wall. ${ }^{17}$

In addition to these energies, there is an energy associated with length distortions of the conventional domain walls, given by

$$
\mathscr{H}_{\mathrm{dw}}=E_{s} \sum_{n} \sqrt{1+\left(\partial_{y} u_{n}\right)^{2}} d y,
$$

where the conventional domain-wall energy (per unit layer) is given by ${ }^{17}$

$$
E_{s}=2 \sqrt{2 \Gamma \lambda_{\text {eff }}} \text {. }
$$

The expansion of the sum of $\mathscr{H}_{\text {dip }}$ and $\mathscr{H}_{\mathrm{dw}}$ to second order in the displacements gives the elastic energy. A detailed calculation of the elastic coefficients is given in Appendixes $A$ and $B$.

\section{THE SEQUENCE OF PHASES AT $H=0$}

In what follows, we present the appropriate continuum energy density, and then we discuss its consequences for long-wavelength fluctuations and topological excitations.

The BKT transition temperature is determined by the elastic moduli. Certain of these have already been studied in Refs. 1 and 2, starting from the microscopic Ham- 
iltonian (see Appendix A), and expanding in terms of long-wavelength displacements.

\section{A. Low $T$, bending energy $\gg$ stripe orientation energy}

When the bending energy greatly exceeds the stripe orientation energy, as can occur for short-wavelength variations of the displacement $u$ along the stripe direction (where the characteristic length $l_{s}$ determining "short" wavelength will be discussed in the next subsection), the macroscopic energy per unit area takes the form

$$
E_{1}=\frac{1}{2} K\left(\partial_{x} u\right)^{2}+\frac{1}{2} \mu\left(\partial_{y}^{2} u\right)^{2},
$$

where for $H=0$ the compression constant $K$ and the bending constant $\mu$ already have been determined in Refs. 1 and 2. This energy density permits longwavelength thermal fluctuations that, without a longwavelength cutoff (of order $l_{s}^{-1}$ ), would make the fluctuations $\left\langle(\delta u)^{2}\right\rangle \sim T \int d k_{x} d k_{y}\left(K k_{x}^{2}+\mu k_{y}^{4}\right)^{-1}$ become infinite, causing the crystal phase to lose its compressional rigidity. ${ }^{1,2}$ In addition, it permits finite-energy dislocations in the stripe-domain structure of energy $E_{d} \sim \Omega L$ and core size $\sqrt{\mu / K} \sim L$. In principle, these dislocations can cause exponentially decaying order, with a characteristic decorrelation length $L \exp (\Omega L / T)$.

\section{B. Low $T$, stripe orientation energy $\geq$ bending energy}

For long-wavelength variations of $u$ along the stripe direction, the total effective energy per unit area takes the form

$$
E_{2}=\frac{1}{2} K\left(\partial_{x} u\right)^{2}+\frac{1}{2} v\left(\partial_{y} u\right)^{2}+\frac{1}{2} \mu\left(\partial_{y}^{2} u\right)^{2} .
$$

The stripe orientation constant $v$ is due to fourth-order gradients in the exchange energy within the conventional domain walls. ${ }^{1,2}$ The characteristic length scale $l_{s}$ (the long-wavelength cutoff of the previous subsection) is

$$
l_{s}=\sqrt{\mu / v} .
$$

It has been previously established ${ }^{1,2}$ that

$$
\mu \sim \Omega L, \quad v \sim \Gamma a^{2} / L l^{3},
$$

where $\Gamma$ is a microscopic exchange constant, $a$ is a microscopic length, and $l$ is the conventional domain-wall thickness, given by Eq. (12), so $l / a \sim\left(\Gamma / \lambda_{\text {eff }} a^{2}\right)^{1 / 2}$. Thus

$$
l_{s} \sim L \sqrt{\Omega a / \Gamma(l / a)^{3}} \sim L \sqrt{\Omega / \lambda_{\mathrm{eff}} a\left(\Gamma / a^{2} / \lambda_{\mathrm{eff}}\right)^{1 / 2}},
$$

which is comparable to or larger than $L$, since $\Omega / \lambda_{\text {eff }} a$ is on the order of unity, and $\left(\Gamma / a^{2}\right) / \lambda_{\text {eff }}$ is on the order of $10^{2}$. For wavelengths shorter than $l_{s}, E_{2}$ approaches $E_{1}$. For wavelengths longer than $l_{s}$, the last term in Eq. (16) can be neglected, so $E_{2}$ approaches $E_{2}^{\prime}$, where

$$
E_{2}^{\prime}=\frac{1}{2} K\left(\partial_{x} u\right)^{2}+\frac{1}{2} v\left(\partial_{y} u\right)^{2}
$$

As shown by Berezinskii, ${ }^{12}$ Mermin, ${ }^{18}$ and Jancovici, ${ }^{19}$ for $d=2$ systems with this type of energy density the long-wavelength thermal fluctuations cause the order parameter $\langle\exp i[u(\mathbf{r})-u(0)]\rangle=\exp \left(-\frac{1}{2}\left[\left\langle u(\mathbf{r}) u(0)-u(0)^{2}\right\rangle\right]\right)$,

to decorrelate algebraically. Hence the system remains, at least locally, a 2D smecticlike crystal at low temperatures.

\section{Transition to the Ising nematic phase}

On the longer distance scale described by $E_{2}^{\prime}$, the 2D smecticlike crystal phase supports dislocations in the stripe-domain structure, now involving compression and stripe reorientation. Because $E_{2}^{\prime}$ has the same structure as for superfluids, and because dislocations are analogous to vortices (where the phase change of $2 \pi$ is replaced by the Burgers vector $2 L$ ), the energy of an individual dislocation is infinite but dislocation pairs of opposite signs have a finite energy (as for individual and bound vortices in superfluids). These lead to a BKT-like transition at $T_{P}$ to an Ising nematic phase, where ${ }^{13}$

$$
T_{P}=\frac{1}{2 \pi} \sqrt{K_{R} v_{R}} L^{2} .
$$

Here, $K_{R}$ and $v_{R}$ are the large-scale limits of the constants $K$ and $v$, which are renormalized due to smectic fluctuations. ${ }^{20,21}$ This equation is obtained from the usual Kosterlitz-Thouless form $K^{\prime} b^{2} / 8 \pi$, where the effective elastic constant $K^{\prime}$ is $\sqrt{K_{R} v_{R}}$ and the Burger's vector $b$ is $2 L .^{22}$

Above $T_{P}$, in the Ising nematic phase, no $x$ derivatives of $u$ appear in the energy density, because thermal dislocations destroy even algebraic long-range order, and make the value of $u$ have no meaning. Thus, the compression constant $K_{R}$ becomes zero. This result also can be derived by the following argument. First, scale the coordinates so that the energy $E_{2}^{\prime}$ becomes isotropic with the common elastic constant $\sqrt{K_{R} v_{R}}$. According to the BKT theory this common elastic constant becomes zero at $T>T_{P}$. Since $v_{R}$ is determined by the properties of each conventional domain wall, it cannot be zero for $T>T_{P}$. Hence we conclude that, if $\sqrt{K_{R} v_{R}}=0$ for $T>T_{P}$, we must have $K_{R}=0$.

Despite the fact that the value of $u$ itself has no meaning, because the stripe orientation constant $v_{R}$ is nonzero the orientation $\theta \approx \partial_{y} u$ retains its physical significance. Using the full form for the stripe orientation energy, the elastic energy per unit area (in notation where the subscript $R$ is suppressed) takes the form

$$
E_{3}=\frac{1}{2} \kappa\left(\partial_{x^{\prime}} \theta\right)^{2}+\frac{1}{2} \mu\left(\partial_{y^{\prime}} \theta\right)^{2}+\frac{1}{16} v[1-\cos 4 \theta] .
$$

Here $x^{\prime}$ and $y^{\prime}$ are local coordinates perpendicular and parallel to the stripes, respectively. The value for $\mu$ is the same as in $E_{1} \cdot 1,2,23$ For small $\theta$, the last term reduces, up to a constant, to the form $(v / 2)\left(\partial_{y} u\right)^{2}$ that was used in $E_{2}$. Over large distances, the stripe orientation energy tends to orient the stripes along either of two mutually perpendicular axes.

In a fixed frame of reference the energy $E_{3}$ reads

$$
\begin{aligned}
E_{3}= & \frac{1}{2} \kappa\left(-\sin \theta \partial_{x} \theta+\cos \theta \partial_{y} \theta\right)^{2}+\frac{1}{2} \mu\left(\cos \theta \partial_{x} \theta\right. \\
& \left.+\sin \theta \partial_{y} \theta\right)^{2}+\frac{1}{16} v[1-\cos 4 \theta] .
\end{aligned}
$$


In Appendix A we calculate the mean-field value for $\kappa$, finding it to be negative:

$$
\kappa_{\mathrm{mf}}=-\frac{7 \Omega L}{4 \pi^{3}} \zeta(3) \text {. }
$$

This indicates, for $T>T_{P}$, an instability toward nonuniform along $x^{\prime}$ tilt angles. In addition, in Appendix C, we calculate the leading contribution of the smectic fluctuations to $\kappa$. This is dominated by the low momentum fluctuations, and requires an infrared cutoff. It provides a contribution to $\kappa$ that is positive and proportional to $T$.

In writing down the mean-field and the low momentum fluctuation contributions, the effects of strong smectic fluctuations at high momentum must be accounted for. Thus, with subscript $R$ for these strongly renormalized quantities, by (C12) we have

$$
\kappa=\left(\kappa_{\mathrm{mf}}\right)_{R}-\frac{T}{64 \pi}\left[\frac{K_{R}}{v_{R}}\right]^{3 / 2} \ln \frac{p_{x}^{2} \mu_{R}}{4 v_{R}},
$$

where the external momentum $p_{x}$ is the infrared cutoff. Here we employ the relations ${ }^{1,2,24}$

$K_{R}=Z^{2} K, \quad v_{R}=Z^{-1} \nu, \quad \mu_{R}=Z^{-1} \mu, \quad\left(\kappa_{\mathrm{mf}}\right)_{R}=Z^{2} \kappa_{\mathrm{mf}}$,

$Z=\left(w^{*}\right)^{2 / 5}\left(\frac{K T^{2}}{\mu^{3}}\right]^{-1 / 5} q_{u v}^{2 / 5}, w^{*}=\frac{64 \pi}{5}$,

where the ultraviolet cutoff is given by

$$
q_{u v}=l_{s}^{-1}=\left(\frac{v_{R}}{\mu_{R}}\right)^{1 / 2}=\left[\frac{v}{\mu}\right]^{1 / 2} .
$$

We determine that, for $T=T_{P}$,

$$
Z=\left[\frac{56 \zeta(3)}{5}\right]^{2 / 11} \approx 1.60
$$

This means that, without the logarithm, the second term in $\kappa$ is about $0.015 K / v$ of the first term. [In our evaluations, we have employed the mean-field values for $K$ and $\mu$, given in (A24) and (A25); $v$ disappears from the expression for $Z$.] Since $K / v \sim(\Gamma / \Omega a)^{1 / 2} \approx 30$, the coefficient of the second term is about half of the first. If the logarithm gives a factor somewhat larger than two, the second term alone might be enough to stabilize the system.

However, there is yet another contribution to $\kappa$ that tends toward stability. It arises only in the Ising nematic phase, where there is a need for such a stabilizing term. In this phase there is an equilibrium density $N$ of free dislocations of both signs, which make a contribution to $\kappa$ that we call $\kappa_{d}$. A semiquantitative estimate, which we now present, indicates that $\kappa_{d}$ is sufficiently large that it alone can overcome the negative mean-field contribution, thus stabilizing the system.

For $T>T_{P}$, the free dislocations form a neutral plasma of dislocations in which the field of an excess dislocation is screened over a finite distance $L_{s}$. To obtain $L_{s}$, we assume Boltzmann statistics for the dislocations, and thus we may employ the 2D Debye screening length, or

$$
L_{s}^{-2}=\frac{2 \pi N_{d} e^{2}}{T},
$$

where $N_{d}$ is the dislocation density and the coupling energy $e^{2}$ is the charge squared. For $e^{2}$ we consider the coefficient of the logarithm in the interaction of two dislocations, thus obtaining for the charge squared [cf. Eq. (21)]

$$
e^{2}=\frac{\sqrt{K v} L^{2}}{2 \pi} \text {. }
$$

For $N_{d}$ we employ the results of Kosterlitz for the plasma phase, ${ }^{13}$ now with characteristic minimal separation $L$, so

$$
N_{d}=L^{-2} \exp \left[-2 b \sqrt{T_{P} /\left(T-T_{P}\right)}\right],
$$

where $T_{P}$ is given in Eq. ( 21$)$ and $b \approx 1.5 .^{13}$ Thus we obtain

$$
L_{s} \sim L \sqrt{T / T_{P}} \exp \left[b \sqrt{T_{P} /\left(T-T_{P}\right)}\right] .
$$

The excess density of dislocations of one sign, $\delta N_{d}$, creates a gradient of stripe orientation angle $\theta$ according to $\partial_{x} \theta \sim L\left(\delta N_{d}\right) \ln \left(L_{s} / L\right)$. (Note that it is linear in $\delta N_{d}$ and, for fixed number of dislocations, inversely proportional to $L$. The logarithmic factor is due to a screening effect that takes place for distances larger than $L_{s}$; the distortion grows as the screening length grows.) This requires an excess free energy per unit area that is quadratic in $\delta N_{d}$ :

$$
\delta E \sim E_{d}\left(\delta N_{d}\right)^{2} / N_{d} \sim E_{d}\left(\partial_{x} \theta\right)^{2} /\left[N_{d}\left(L \ln \left(L_{s} / L\right)\right)^{2}\right] .
$$

The corresponding contribution $\kappa_{d}$ to the elastic coefficient is thus on the order of $E_{d} /$ $\left[N_{d}\left(L \ln \left(L_{s} / L\right)\right)^{2}\right]$. An estimate of $E_{d}=\Omega L$ $\sim 2 T \ln \left(1 / \sqrt{N_{d}} L\right)$ can be obtained from the work of Kosterlitz and Thouless. ${ }^{13}$ Thus we estimate that

$$
\kappa_{d} \approx T /\left[N_{d}\left(L \ln \left(L_{s} / L\right)\right)^{2}\right] .
$$

(Note that $N_{d} L^{2}$ must be small, or no greater than 1, for this to be valid.) Hence, for a rather high concentration of dislocations (e.g., $N_{d} L^{2}=0.1$ ), $\kappa_{d} \approx 10 T$. We therefore conclude that the most probable situation is that, within the Ising nematic phase, the overall $\kappa$ is positive.

Nevertheless, we still cannot exclude the possibility of negative $\kappa$. If it is negative, the Hamiltonian of elastic distortion, Eq. (23), favors the development of textures with large gradients. The characteristic size of such a texture is defined by higher derivative terms in the elastic energy not included in Eq. (23). They become relevant at distances of the order of magnitude of $1 / \sqrt{N_{d}}$ or $L$. Over such distances elastic theory becomes inapplicable; moreover, over this distance scale the fluctuations in the density of dislocations is very large. Therefore the system should be considered to be completely random over this distance scale.

To summarize, according to the sign of $\kappa$, two possibilities arise for the phase diagram when $T>T_{P}$ :

(1) When $\kappa<0$, we expect that melting will be via a first-order phase transition to a liquid of domains (with either of two mutually perpendicular orientations) pos- 
sessing local nematic order but global tetragonal order. Because there are two preferred stripe orientations, the stripe domains are still preferentially oriented along either of two mutually perpendicular directions. In contrast to the Ising nematic phase, in this phase the probabilities for both directions are equal. For this reason we call it a tetragonal liquid. This phase persists until the spin reorientation phase transition.

(2) When $\kappa>0$, the system undergoes a KT-like transition at $T_{P}$ from a 2D smecticlike crystal phase to one with Ising nematic order. At a higher temperature $T_{O}$, this Ising nematic system melts to the tetragonal liquid phase, with local nematic order but global tetragonal order. This phase persists until the spin reorientation phase transition.

\section{ISING NEMATIC AND TETRAGONAL LIQUID PHASE}

A transition in 2D from a positionally and orientationally ordered crystal to one possessing only orientational order (the hexatic phase, with sixfold orientational order) was considered by Halperin and Nelson. ${ }^{25}$ The present system of stripe-domain walls, with its exchange-induced stripe orientation anisotropy, differs from the free 2D crystal they considered: a free-standing 2D crystal can be given an arbitrary rotation or translation without change of energy, whereas in the present case the stripe domains have a preferential orientation with respect to the crystal lattice of the magnetic field and its substrate. The present system is somewhat like a nematic in an applied electric or magnetic field, which tends to orient the nematic with twofold symmetry, but gives it no positional order. However, the present crystal field has fourfold (tetragonal) symmetry. Moreover, because the system has no long-range positional order, the fourfold symmetry reduces to twofold symmetry, and thus we call it the Ising nematic phase.

The orientational order parameter $\eta$ is given by

$$
\eta=\exp (i 2 \theta) \text {. }
$$

It has a nonzero thermodynamic average $\langle\eta\rangle$ for $T>T_{P}$. For $\kappa>0$, it is governed by the energy density $E_{3}$, which has $Z_{2}$ (Ising-like) symmetry with respect to the order parameter. Thus, we expect that long-range orientational order persists for $T_{P}<T<T_{O}$, and that the phase transition at $T_{O}$ is in the Onsager-Ising universality class. In particular, we expect that $\langle\eta\rangle \sim\left(T_{O}-T\right)^{1 / 8}$ just below the orientational melting point $T_{O}$.

Employing the angle $\theta$, the total energy in the Ising nematic phase (IN) takes, from Eq. (23), the form

$$
\begin{aligned}
E_{\mathrm{IN}}= & \frac{1}{2}(\kappa+\mu)(\nabla \theta)^{2} \\
& +\frac{1}{2}(\kappa-\mu)\left\{\cos 2 \theta\left[\left(\partial_{x} \theta\right)^{2}-\left(\partial_{y} \theta\right)^{2}\right]\right. \\
& \left.\quad+\sin 2 \theta\left(2 \partial_{x} \theta \partial_{y} \theta\right)\right\} \\
& +\frac{v}{16}(1-\cos 4 \theta) .
\end{aligned}
$$

We first neglect the stripe orientation anisotropy constant $v$, which operates only over relatively large distances. Moreover, the terms in $\cos 2 \theta$ and $\sin 2 \theta$ renormalize to zero over large distances (up to the distance scale $l_{s}$ determined by $v$ ) when the thermally induced long-wavelength fluctuations are included. Thus the effective elastic constant is $\kappa+\mu$. We now estimate the Ising-like transition temperature $T_{O}$. To do so, we begin by noting that, as the temperature is increased, there will tend to be a proliferation of disclination-antidisclination pairs. As long as their characteristic separation is shorter than

$$
l_{s}^{\prime}=\sqrt{(\kappa+\mu) / v},
$$

so that the stripe orientation anisotropy may be neglected, these will be like the ordinary variety of disclinationantidisclination pairs, ${ }^{26}$ and would lead to a KosterlitzThouless transition. However, at a temperature just below that at which such a transition would actually occur, the characteristic separation of the disclinationantidisclination pairs becomes comparable to and then, on further increase of temperature larger than $l_{s}^{\prime}$. At that point the stripe orientation anisotropy takes over, changing the nature of the disclination-antidisclination pairs, so that at large distances the stripes become oriented along either of the two favored, mutually perpendicular, directions. The energy of such pairs is proportional to their separation, as with domain walls, and thus we expect these excitations to lead to an Ising-like transition at $T_{O}$. To estimate $T_{O}$, we employ the value associated with the Kosterlitz-Thouless transition that does not quite occur. This leads to

$$
T_{O}=\frac{\pi}{8}(\kappa+\mu) \text {. }
$$

This equation is obtained from the usual KosterlitzThouless form $K b^{2} / 8 \pi$, where $K=\kappa+\mu$ is the elastic constant and $b=\pi$ is the Burgers vector corresponding to an individual disclination.

A new type of topological excitation occurs in the Ising nematic phase, which we call the stripe rotation domain wall. This linear defect separates regions in which the stripes are horizontal from those in which the stripes are vertical. These defects are expected to proliferate just above $T_{O}$. In what follows, we determine the profile of $\theta$, the energy, and the preferential orientation of these stripe rotation domain walls.

Let the stripe rotation domain wall be tilted with respect to the $x$ axis by the angle $\alpha$. This means that the function $\theta(x, y)$ that minimizes Eq. (36) is a function of $\xi=x \cos \alpha+y \sin \alpha$. Because of the fourfold symmetry of the system, it will be sufficient to consider the case where $\theta$ takes on the value 0 for $\xi \rightarrow-\infty$, and either of $\pm \pi / 2$ for $\xi \rightarrow+\infty$. The energy density becomes

$$
E_{\mathrm{IN}}^{\prime}=g(\theta)\left(\partial_{\xi} \theta\right)^{2}+h(\theta),
$$

where

$$
\begin{aligned}
& g(\theta)=\frac{1}{2}[(\kappa+\mu)+(\kappa-\mu) \cos 2(\theta-\alpha)], \\
& h(\theta)=\frac{v}{16}(1-\cos 4 \theta),
\end{aligned}
$$


This is minimized by

$$
0=\partial_{\theta}\left[h(\theta)-g(\theta)\left(\partial_{\xi} \theta\right)^{2}\right] .
$$

In the present case the constant of integration is zero, since $h(\theta) \rightarrow 0$ and $\partial_{\xi} \theta \rightarrow 0$ as $\xi \rightarrow \infty$. Hence

$$
\partial_{\xi} \theta= \pm \sqrt{h(\theta) / g(\theta)},
$$

whose solution is given by

$$
\xi= \pm \int_{\theta_{0}}^{\theta} \sqrt{g(\theta) / h(\theta)} d \theta
$$

For our particular case,

$$
\begin{aligned}
\xi & = \pm \int_{\theta_{0}}^{\theta} \sqrt{g(\theta) / h(\theta)} d \theta \\
& = \pm l_{s}^{\prime} \int_{\theta_{0}}^{\theta} \frac{\sqrt{1+\sigma \cos 2(\theta-\alpha)}}{\sin 2 \theta} d \theta,
\end{aligned}
$$

where $\sigma=(\kappa-\mu) /(\kappa+\mu)$, and $\theta_{0}$ will be discussed shortly. This is a quadrature. Note the appearance of the characteristic length $l_{s}^{\prime}$.

The energy per unit length is given by

$$
E_{O}=\int E_{\mathrm{IN}}^{\prime} d \xi=2 \int \sqrt{g(\theta) h(\theta)} d \xi .
$$

For our particular case,

$E_{O}= \pm \frac{\sqrt{(\kappa+\mu) v}}{2} \int_{0}^{ \pm \pi / 2} \sin 2 \theta \sqrt{1+\sigma \cos 2(\theta-\alpha)} d \theta$

where both +'s are paired and both -'s are paired. This expression must be minimized as a function of $\alpha$. There are two types of stripe rotation domain walls within this framework, the appropriate choice depending upon the sign of $\sigma$. We discuss a number of cases:

(a) For $\sigma<0$ and $\theta_{\infty}=\pi / 2$, we have determined numerically that $\alpha=\pi / 4$ minimizes $E_{O}$. In this case the stripe rotation domain wall is given in Fig. 3(a). Note

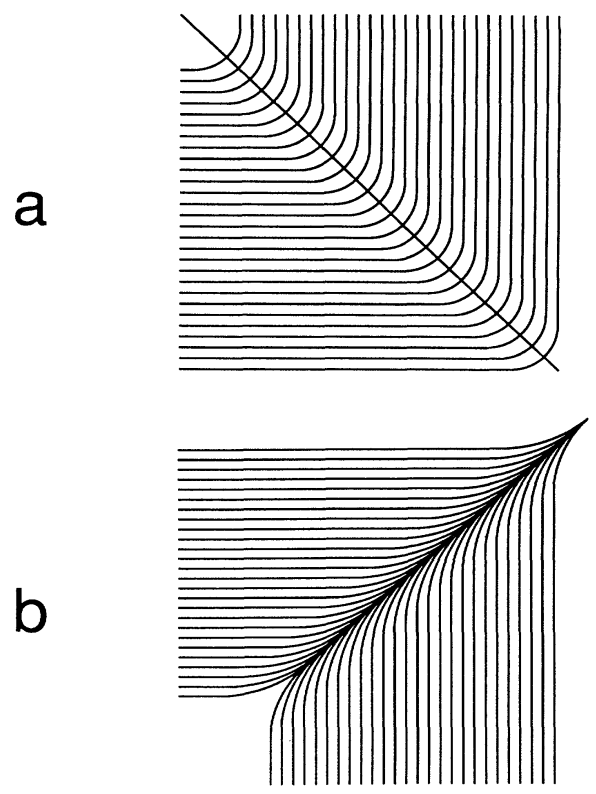

FIG. 3. Two stripe rotation domain-wall configurations. that for $\alpha=\pi / 4$ the second term in the square root of Eq. (46) reduces to $\sigma \sin 2 \theta$, so the square root has its minimum when $\sin \theta$ has its maximum.

(b) For $\sigma>0$ and $\theta_{\infty}=\pi / 2$, we have determined numerically that $\alpha=3 \pi / 4$ minimizes $E_{O}$. In this case the stripe rotation domain wall is given in Fig. 3(b). Note that for $\alpha=3 \pi / 4$, the second term in the square root of Eq. (46) reduces to $-\sigma \sin 2 \theta$, so the square root has its minimum when $\sin \theta$ has its maximum. (Despite the appearance of Fig. 3(b), it is not a representation of the stripe density, but rather only of their orientation: their density does not become infinite at the stripe rotation domain wall.)

(c) For $\sigma>0$ and $\theta_{\infty}=-\pi / 2, \alpha=\pi / 4$ minimizes $E_{O}$. In this case the stripe rotation domain wall is a rotated version of Fig. 3(b).

(d) For $\sigma<0$ and $\theta_{\infty}=-\pi / 2, \alpha=3 \pi / 4$ minimizes $E_{O}$. In this case the stripe rotation domain wall is a rotated version of Fig. 3(a).

We thus come to the conclusion that, by simple visual inspection of the stripes in a given sample, one can determine the sign of $\sigma=(\kappa-\mu) /(\kappa+\mu)$, and thus find which of $\kappa$ and $\mu$ is larger. If the stripes simply appear to bend, as in Fig. 3(a), then $\sigma<0$, or $\kappa<\mu$, which is reasonable if $\kappa$ is only slightly positive because it involves cancelling positive and negative terms.

The intersection of two stripe rotation domain walls, which are perpendicular to one another, is a wedgelike structure with a pointlike singularity. This singularity is precisely the large scale (distances large than $l_{s}^{\prime}$ ) version of an ordinary disclination, and within $l_{s}^{\prime}$ of the point singularity this structure looks like a standard disclination. However, at larger distances the stripes are oriented along either of the two favored, mutually perpendicular, directions. The energy of such a disclination is proportional to the wall length.

Consider now domain formation (e.g., of vertical stripe orientation within a bulk system of horizontal stripe orientation). If the domain is of dimension larger than the characteristic domain wall thickness $l_{s}^{\prime}$, the orientation energy will cause it to have a rectangular shape, with sides making angles of $\pi / 4$ and $3 \pi / 4$ to the horizontal. At each corner there will be a pointlike singularity, as discussed in the previous paragraph, with characteristic kink energy

$$
E_{K} \sim E_{O} l_{s}^{\prime} \sim(\kappa+\mu) .
$$

The energy to form the domain will be the sum of the wall energy, given by the product of $E_{O}$ times the perimeter, and of the kink energy, given by the product of $E_{K}$ times the number of kinks.

For $T>T_{O}$, both types of stripe orientations can occur, giving the system the symmetry of a tetragonal liquid with an external ordering field.

\section{KOSTERLITZ-THOULESS TRANSITION $T_{P}$ IN A FIELD}

Equation (21) for $T_{P}$ requires that the compression constant $K$ be obtained in a field. We have performed a 
formal evaluation in Appendix B, but it can also be obtained by a physical argument. To do so we recall a number of results from Ref. 2.

The reduced magnetization (multiply by $g \mu_{B} S / a^{3}$ to get the true magnetization), is given by

$$
m=\frac{(L+\delta)-(L-\delta)}{(L+\delta)+(L-\delta)}=\frac{\delta}{L}=n \delta
$$

and the energy, per unit area and per unit layer, is given by

$$
\begin{aligned}
& E=E_{s} n-\frac{\Omega}{\pi} n \ln \left[\frac{2}{\pi n l} \cos \frac{\pi n \delta}{2}\right]-h n \delta, \quad n \equiv \frac{1}{L}, \\
& h \equiv g \mu_{B} S H / a^{2} .
\end{aligned}
$$

Equation (48) is valid if $L-\delta \gg l$.

Minimization with respect to both $n$ and $\delta$, as done in Ref. 2, leads to the equilibrium conditions

$$
\delta_{0}=\frac{2}{\pi n_{0}} \sin ^{-1} \frac{h}{h_{c}}, \quad n_{0}=n^{*}(T) \sqrt{1-\left(h / h_{c}\right)^{2}},
$$

where

$$
n^{*}(T)=\frac{2}{\pi l} \exp \left[-\frac{\pi E_{s}}{\Omega}-1\right], \quad h_{c} \equiv \Omega n^{*}(T) / 2 .
$$

Thus the mean field magnetization is

$$
m_{0}=n_{0} \delta_{0}=\frac{2}{\pi} \sin ^{-1} \frac{h}{h_{c}} .
$$

It is convenient to consider the energy to be a function of both $n$ and $m=n \delta$. Expanding it to second order in derivations from equilibrium, we find

$\delta E=\frac{E_{n n}}{2}(\delta n)^{2}+\frac{E_{m m}}{2}(\delta m)^{2}+E_{n m}(\delta n)(\delta m)$,

where

$$
\begin{aligned}
& E_{n n} \equiv \frac{\partial^{2} E}{\partial n^{2}}=\frac{\Omega}{\pi n}, \quad E_{m m} \equiv \frac{\partial^{2} E}{\partial m^{2}}=\frac{\pi \Omega n}{4} \sec ^{2} \frac{\pi m}{2}, \\
& E_{n m} \equiv \frac{\partial^{2} E}{\partial n \partial m}=\frac{\Omega}{2} \tan \frac{\pi m}{2} .
\end{aligned}
$$

We now consider $n$ to have a specific value, and we thermally average (52) over the variable $m$. Up to a constant that arises from the thermal averaging, we then may rewrite Eq. (52) as

$$
\delta E=\frac{K_{\mathrm{eff}}}{2}\left[\frac{\delta n}{n}\right]^{2},
$$

where the effective compression constant is given explicitly by

$$
K_{\mathrm{eff}}=n^{2}\left(E_{n n}-E_{n m}^{2} / E_{m m}\right)=\frac{\Omega n^{*}}{\pi}\left[1-\frac{h^{2}}{h_{c}^{2}}\right]^{3 / 2} .
$$

The choice of $m$ as the second variable is not essential to this argument. It was only necessary that a specific choice of second variable, independent of $n$, be taken. For example, use of the variables $n$ and $\delta$ would yield the same result for $K_{\text {eff }}$ because, in local equilibrium, where first derivatives are all zero, $E_{n n}-E_{n \delta}^{2} / E_{\delta \delta}=E_{n n}$ $-E_{n m}^{2} / E_{m m}$. [In this expression, on the left-hand-side we write $E(n, \delta)$, whereas on the right-hand-side we write $E(n, m)$.] Appendix B evaluates $K_{\text {eff }}$ from a matrix diagonalization, obtaining agreement with the present result.

Use of $K_{\text {eff }}$ and $v$ in Eq. (21) leads to

$$
T_{P}=\frac{\sqrt{K_{\mathrm{eff}} v}}{2 \pi} L^{2} \sim \frac{1}{n^{*}} \sqrt{\Omega \Gamma a^{2} / l^{3}} .
$$

Thus $T_{P}$ is essentially independent of field, and this equation becomes a self-consistent equation for $T_{P}$. Its dominant temperature dependence arises from $n^{*}$. For $\Omega a \approx 1 \mathrm{~K}, \Gamma \approx 1000 \mathrm{~K}, l \approx 10 a$, and $1 / n^{*}=L$, we get $T_{P} \approx 10(L / l) K$. At low $T$ there is no solution, but near the spin reorientation transition $T_{R}$, where $L$ decreases and $l$ increases, there is a solution. Hence, within the stripe-domain phase, there should be a transition from a smectic-like crystal phase to an Ising nematic phase with short-range smecticlike order.

Including the effect of renormalization on $K_{\text {eff }}$ and $v$ produces only small corrections to $T_{P}(H)$.

\section{PHASE DIAGRAM IN $\left(H_{1}, H_{\|}, T\right)$ SPACE}

Here we describe the global phase diagram of a thin ferromagnetic film in the three-dimensional space given by $H_{\|}, H_{\perp}$, and $T$, where $H_{\|}$and $H_{\perp}$ are the components of the field parallel and perpendicular to the plane. (Previously we have considered only $H_{\perp}$.) It is depicted in Fig. 4. It is based upon an extrapolation from ordering of phases for $H=0$, and is expected to be correct in the topological sense. It is quite likely that experimental results will deviate in a quantitative way from this phase di-

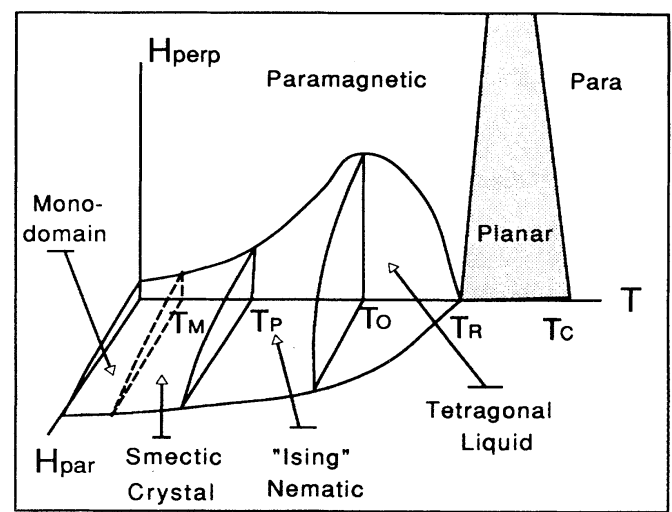

FIG. 4. Phase diagram when $\kappa>0$, where the Ising nematic is stable. Note that the scale for $H_{\|}$and the maximum value for $H_{\perp}$ in the planar phase is on the order of the spin anisotropy energy $(\sim 100 \mathrm{Oe})$, whereas the scale for $H_{\perp}$ in the stripe-domain phases is on the order of an Oe. All the phase transitions except $T_{c}$ are much closer to $T_{R}$ than is shown in the figure. 
agram, and it is not inconceivable that there will be significant deviations from it. Experiment, of course, will yield the phase diagram for an actual system. As discussed below, real systems will appear to have a somewhat different phase diagram, also depicted in Fig. 4, but with the monodomain phase considered to be the same as the paramagnetic phase.

The local order of the stripe-domain structure can exist in three modifications distinguished by their differing long-range order: the smectic phase, the Ising nematic phase, and the tetragonal liquid phase. In each of these phases, the characteristic $H_{\perp}$ at which the system becomes paramagnetic is determined by $h_{c}$ of Eq. (50).

At sufficiently low temperatures, the stripe-domain width $L$ is sufficiently large that a single domain fills a real sample, thus giving the impression of a ferromagnetic state that is continuously connected to the paramagnetic state. However, for purposes of representing the phase diagram, we must consider the thermodynamic limit where the sample size is infinite. Thus an infinite sample will contain an infinite number of domains. In the true stripe-domain phase, there is long-range orientational order but, due to long-wavelength fluctuations, there is algebraically decaying positional order. This phase supports bound pairs of dislocations with equal and opposite Burgers' vectors. Adding $H_{\perp}$ favors the growth of the domain whose spins are oriented along the field, so that the temperature at which domains of one spin direction fill all of the area should decrease with increasing field. Adding $H_{\|}$does not affect either type of domain to lowest order in the field, so we have drawn this smectic-nematic phase separation line to have no dependence on $H_{\|}$.

On further increase of temperature, to $T_{P}$, unbound dislocations proliferate, causing there to be exponentially decaying positional order. However, it retains long-range stripe orientation order. This phase may be described as an Ising nematic in which the conventional domain walls are oriented along one of two mutually perpendicular directions determined by the underlying substrate. Regions of such mutually perpendicular stripe directions are separated by what we call a stripe rotation domain wall. We have shown that $T_{P}$ is independent of $H_{\perp}$. Presently we have no clear idea of what happens when $H_{\|}$is added, so we draw this phase separation line to have no dependence on $H_{\|}$.

At the even higher temperature $T_{o}$, the tetragonal symmetry is restored in the third order phase, in which the stripe rotation domain walls proliferate. We have called this phase a tetragonal liquid because there is exponentially rapid spatial decorrelation from one stripe orientation to the other. Adding $H_{\|}$should have no obvious effect on the stability of this phase relative to the Ising nematic phase.

For temperatures above $T_{R}$ the system is in the planar phase, which has no domain structure. In the absence of in-plane spin anisotropy, the planar phase is strictly a plane in the three-dimensional space, much as the ferromagnetic phase for an Ising model is the line $H=0$ in $(H, T)$ space. If the in-plane spin anisotropy is not zero, the planar phase exists in a range of $H_{\perp}$ determined by the in-plane spin anisotropy.

Note that, near $T_{R}$, with $T<T_{R}$, the average domain separation $L$ saturates at a value on the order of the dipole length of Eq. (7), as discussed by Yafet and Gyorgy. ${ }^{27}$ See also Ref. 2.

The phase transition from the stripe-domain crystal to the Ising nematic is most probably in the BerezinskiiKosterlitz-Thouless class (i.e., dislocation-mediated melting). The transition from Ising nematic to tetragonal liquid is most probably in the Ising class (i.e., stripe rotation domain-wall mediated melting). The universality class of the transition from tetragonal liquid to planar phase has not yet been investigated.

We emphasize that the scales of the magnetic fields at the transition lines vary significantly. For the stripedomain phases in $H_{1}$, on the transition lines the scale for $H_{\perp}$ is given via Eq. (50), with $H_{c}=h_{c}\left(a^{2} / g \mu_{B} S\right)$, a value determined by the ratio of the dipolar energy to the magnetization per unit length of a stripe. It contains the large stripe width in the denominator, and thus is relatively small, on the order of $1 \mathrm{Oe}$. On the other hand, for the stripe-domain phases in $H_{\|}$, on the transition lines the scale for $H_{\|}$is determined by the effective spin anisotropy $\lambda_{\text {eff }}$, which is on the order of 1000 Oe. The same large estimate applies for $H_{\perp}$ in the planar phase, since the magnetic field must pull the magnetization out of the plane against the spin anisotropy. Note that even for the stripe-domain phases $H_{\perp}$ varies by many orders of magnitude, being on the order of $\Omega / l$ at which temperatures, but on the order of $\Omega / L$ at low temperatures, where $L / l$ is exponentially large. Thus $H_{\perp}$ is on the order of hundredths of an Oersted or less at low temperatures.

The phase diagram for a finite film differs from that for an infinite sample in two respects. First, for a finite sample, at low temperatures the monodomain phase of a finite sample cannot be distinguished from the paramagnetic phase. Second, the stripe size decreases so rapidly

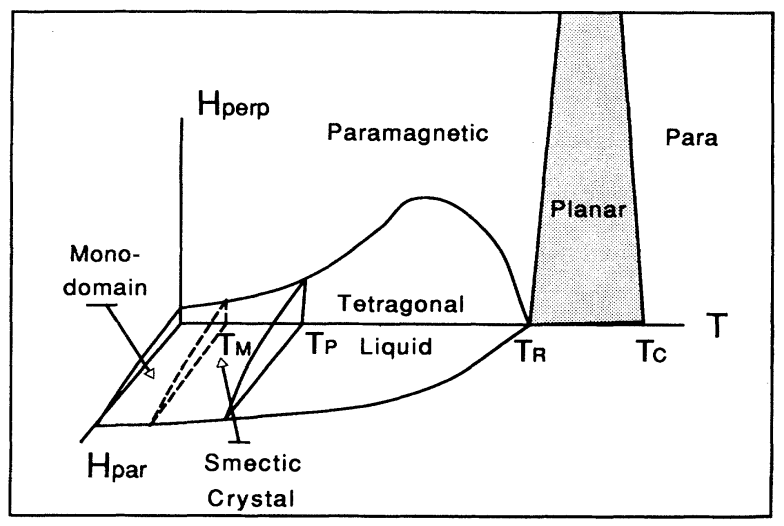

FIG. 5. Phase diagram when $\kappa<0$, where the Ising nematic is unstable. Note that the scale for $H_{\|}$and the maximum value for $H_{\perp}$ in the planar phase is on the order of the spin anisotropy energy $(\sim 100 \mathrm{Oe})$, whereas the scale for $H_{1}$ in the stripedomain phases is on the order of an Oe. All the phase transitions except $T_{c}$ are much closer to $T_{R}$ than is shown in the figure. 
with increasing temperature that the transition from a single domain to the stripe-domain phase mimics a true phase transition. For that reason we indicate it by a dashed line, reserving solid lines for true phase transitions.

Finally, in Fig. 5, we present the phase diagram as it would appear for a finite film if the Ising nematic phase is unstable. Note that the phase transition between the smectic phase and the tetragonal liquid is expected to be first-order.

\section{SUMMARY AND CONCLUSIONS}

We have studied the properties of ferromagnetic thin films that are subject to what has been called the spin reorientation transition. We predict a number of new results, including two new phases based upon a local stripe-domain structure. One is the Ising nematic phase, in which the tetragonal symmetry is reduced to $Z_{2}$ Ising symmetry. The other is the tetragonally symmetric liquid of mutually perpendicular stripe domains.

The Ising nematic phase supports long-range stripe orientation order. The transition between these two new phases is mediated by the proliferation of a new type of topological defect, a stripe rotation domain wall that separates regions of two mutually perpendicular orientations. We have found the stripe domain domain-wall structure (i.e., the distribution of orientation), their energy, and their width. We have established the equation for the long-range distortion for the Ising nematic, which is analogous to the Sine-Gordon equation, with fielddependent coefficients of the derivative terms.

We have analyzed the phase transition between the Ising nematic phase and the tetragonal liquid phases, and established that it is in the Ising universality class. We have found that, in the mean-field approximation, the Ising nematic is unstable with respect to orientational nonuniformities normal to the stripe direction. However, this instability can be suppressed by thermal fluctuations. Nevertheless, we cannot exclude the possibility that this instability results in a first-order phase transition from the smectic crystal directly to the tetragonal liquid.

All these phase transitions take place in the vicinity of the spin reorientation phase transition. Therefore, a high degree of resolution in temperature will be needed in order to identify the new phases. We have also found the global phase diagram in the three-dimensional space defined by $\left(H_{\|}, H_{\perp}, T\right)$. It contains the same phases as discussed here, as well as the planar phase and the paramagnetic phase.

All three of the stripe-domain phases are very sensitive to $H_{\perp}$ (the characteristic field is on the order of $1 \mathrm{Oe}$ ), the much less sensitive to $H_{\|}$. Measurements in controlled weak fields would be highly desirable.

We predict the existence of either one or two tricritical points (according to whether $\kappa<0$ or $\kappa>0$ ), and a very special singular point at the spin reorientation transition $T_{R}$ for zero field, at which three singular lines meet. The character of the singular behavior near this point is an open problem.

We have estimated (cf. Appendices A and B) the global stability of each of the new phases under small fluctua- tions. Note that, although these phases were studied in the context of films that undergo the spin reorientation transition, there is no requirement that such a reorientation transition take place. Thus, it is, in principle, possible to have a film of just the right thickness that the perpendicular surface spin anisotropy always dominates, yet $\lambda_{\text {eff }}$ is significantly renormalized, so there will be no spin reorientation transition. In this case, the system can go, with increase of temperature, from a monodomain to a smectic crystal to an Ising nematic to a tetragonal liquid, until the Curie point is reached at the highest of temperatures.

\section{APPENDIX A: STABILITY OF THE STRIPED CRYSTAL AND MEAN-FIELD ELASTIC CONSTANTS FOR $\boldsymbol{H}=0$}

In this appendix we find the change of energy to second order in displacements $u_{n}(y)$ of the domain walls. Performing this expansion on the sum of Eqs. (13) and (8) yields

$$
\begin{aligned}
\delta \mathcal{H}= & \frac{E_{s}}{2} \sum_{n} \int\left[\frac{\partial u_{n}}{\partial y}\right]^{2} d y \\
& -\sum_{m, n}(-1)^{m-n} \iint d y d y^{\prime} V\left(r_{m n}\right) \frac{\partial u_{m}}{\partial y} \frac{\partial u_{n}}{\partial y^{\prime}} \\
& -\frac{1}{2} \sum_{m, n}(-1)^{m-n} \iint d y d y^{\prime} W\left(\mathbf{r}_{m n}\right) \\
& \times\left[u_{m}(y)-u_{n}\left(y^{\prime}\right)\right]^{2},
\end{aligned}
$$

where

$$
\begin{aligned}
& V(r)=\frac{\Omega}{\pi \sqrt{r^{2}+l^{2}}}, \quad W(\mathbf{r})=V^{\prime}(r) \frac{y^{2}}{r^{3}}+V^{\prime \prime}(r) \frac{x^{2}}{r^{2}}, \\
& \mathbf{r}_{m n}=\left[(n-m) L, y-y^{\prime}\right] .
\end{aligned}
$$

The cutoff length $l$ accounts for the finite domain-wall width.

The quadratic form (A1) can be diagonalized by Fourier transformation. We denote by $u_{\mathrm{p}}$ the Fourier transform of $u_{m}(y)$, where $-\pi / L \leq p_{x} \leq \pi / L$ and $-\infty \leq p_{y} \leq \infty$. Then

$$
u(\mathbf{r})=\iint \frac{d^{2} p}{(2 \pi)^{2}} u_{\mathrm{p}} e^{i \mathbf{p} \cdot \mathbf{r}} .
$$

The diagonalization of the quadratic form (A1) can be reduced to the calculation of the Fourier transform $\widehat{V}\left(p_{x}, p_{y}\right)$ of the kernel $V(r)$ and its derivatives. One should also take into account the equilibrium condition

$$
\begin{aligned}
& E_{s}=\sum_{j}(-1)^{j} \int d y\left[V\left(R_{j}\right)-V^{\prime}\left(R_{j}\right) \frac{x_{j}^{2}}{R_{j}}\right], \\
& R^{2}=x_{j}^{2}+y^{2}+l^{2}, \quad x_{j}=j L .
\end{aligned}
$$

Let us introduce the Fourier transform $\widetilde{V}(p)$ of the function $V(r)$, defined for any $x$ and $y$

$$
V(\mathbf{r})=\iint\left(d^{2} p\right) \widetilde{V}(\mathbf{p}) e^{i \mathbf{p} \cdot \mathbf{r}},
$$

where we introduce $\left(d^{2} p\right)=d^{2} p /(2 \pi)^{2}$ for brevity. Keeping in mind later applications to finite magnetic field, we 
calculate the more general Fourier transform $\widehat{V}(\mathbf{p})$ of the function $V(2 j L, y)$ of a discrete variable $x_{j}=2 j L$ and a continuous variable $y$

$$
\begin{aligned}
\widehat{V}(\mathbf{p}) & =\sum_{j} \int d y e^{-2 i p_{x} j L-i p_{y} y} V(2 L j, y) \\
& =\frac{1}{2 L} \sum_{k} \widetilde{V}\left[p_{x}+\frac{\pi k}{L}, p_{y}\right]
\end{aligned}
$$

and the Fourier transform $\hat{V}_{\sim}(\mathbf{p})$ of the function $V[(2 j+\bar{\delta}) L, y]$

$$
\begin{aligned}
\widehat{V}_{\sim}(\mathbf{p}) & =\sum_{j} \int d y V[(2 j+\bar{\delta}) L, y] e^{-2 i p_{x} j L-i p_{y} y} \\
& =\frac{e^{i p_{x} L \bar{\delta}}}{2 L} \sum_{k} e^{i \pi k \bar{\delta}} \widetilde{V}\left[p_{x}+\frac{\pi k}{L}, p_{y}\right],
\end{aligned}
$$

where we introduce the dimensionless variable

$$
\bar{\delta}=\delta / L+1 .
$$

In the last two expressions we have used Eq. (A6) and the Poisson summation formula

$$
\sum_{j=-\infty}^{+\infty} e^{i j p}=2 \pi \sum_{k=-\infty}^{+\infty} \delta(p-2 \pi k)
$$

To find $\widetilde{V}(\mathbf{p})$ we use a Gaussian integral representation of the inverse square root occurring in the kernel $V(r)$

$$
\frac{1}{\sqrt{r^{2}+l^{2}}}=\frac{1}{\sqrt{\pi}} \int_{-\infty}^{\infty} d \xi e^{-\xi^{2}\left(r^{2}+l^{2}\right)}
$$

Then

$$
\begin{aligned}
\widetilde{V}(\mathbf{p}) & =\frac{\Omega}{\pi} \frac{1}{\sqrt{\pi}} \iiint d x d y d \xi e^{-i \mathbf{p} \cdot \mathbf{r}-\xi^{2} r^{2}} \\
& =\frac{\Omega}{\sqrt{\pi}} \int \frac{d \xi}{\xi^{2}} e^{-\xi^{2} l^{2}-p^{2} / 4 \xi^{2}}
\end{aligned}
$$

and the last integration, over $\xi$, leads to

$$
\widetilde{V}(\mathbf{p})=\frac{2 \Omega}{p} e^{-p l} .
$$

The remaining unknowns can be found by the use of Eqs. (A7) and (A8) and the relation

$$
W(\mathbf{x})=\frac{\partial^{2} V(\mathbf{x})}{\partial x^{2}} .
$$

Then

$$
\begin{aligned}
& \widetilde{W}(\mathbf{p})=-\frac{2 \Omega p_{x}^{2}}{p} e^{-p l}, \\
& \widehat{V}_{\sim}(\mathbf{p})=\frac{\Omega}{L} e^{i p_{x} L \bar{\delta}} \sum_{k} e^{i \pi k \bar{\delta}} \frac{e^{-l p_{k}}}{p_{k}}, \\
& \hat{W}_{\sim}(\mathbf{p})=-\frac{\Omega}{L} e^{i p_{x} L \bar{\delta}} \sum_{k} e^{i \pi k \bar{\delta}} \frac{\left(p_{x}+a_{k}\right)^{2} e^{-l p_{k}}}{p_{k}}, \\
& \left.\widehat{V}_{(\mathbf{p})}\right)=\left.\hat{V}_{\sim}(\mathbf{p})\right|_{\bar{\delta}=0}, \quad \widehat{W}(\mathbf{p})=\left.\hat{W}_{\sim}(\mathbf{p})\right|_{\bar{\delta}=0} .
\end{aligned}
$$

In the last three formulas $p_{k}^{2}=\left(p_{x}+a_{k}\right)^{2}+p_{y}^{2}$ and $a_{k}=\pi k / L$.
According to (A1) and the definitions (A7) and (A8)

$$
\begin{aligned}
\delta \mathscr{H}=\frac{1}{L} \int & \left\{p_{y}^{2}\left(\frac{E_{s}}{2}-\widehat{V}(\mathbf{p})+\widetilde{V}_{\sim}(\mathbf{p})\right]\right. \\
& \left.-\left(\hat{W}(0)-\hat{W}_{\sim}(0)-\hat{W}(\mathbf{p})+\hat{W}_{\sim}(\mathbf{p})\right)\right\} \\
& \times u_{\mathrm{p}} u_{-\mathbf{p}}\left(d^{2} p\right),
\end{aligned}
$$

where the argument $\bar{\delta}$ in $\hat{V}_{\sim}(\mathbf{p})$ and $\hat{W}_{\sim}(\mathbf{p})$ is set to 1 . For the equilibrium condition (A5) we obtain

$$
E_{s}=\widetilde{V}(0)-\hat{V}_{\sim}(0)+\left.\frac{\partial}{\partial p_{x}} p_{x}\left(\widehat{V}(\mathbf{p})-\hat{v}_{\sim}(\mathbf{p})\right)\right|_{\mathbf{p}=0} .
$$

Using Eqs. (A16) -(A18) one obtains

$$
\delta H=\frac{1}{2 L} \int\left(d^{2} p\right) \Phi(\mathbf{p}) u_{\mathrm{p}} u_{-\mathrm{p}},
$$

where

$$
\begin{gathered}
\Phi(\mathbf{p})=\frac{2 \Omega}{L^{2}} \sum_{k=-\infty}^{\infty}\left\{e^{-l\left|b_{k}\right|}\left[\frac{p_{y}^{2}}{2} \mid \frac{1}{\left|b_{k}\right|}-l\right]+\left|b_{k}\right|\right] \\
\left.-e^{-l s_{k}} s_{k}\right\},
\end{gathered}
$$

and $b_{k}=(2 k+1) \pi / L, s_{k}^{2}=\left(p_{x}+b_{k}\right)^{2}+p_{y}^{2}$.

In the limit $l \rightarrow 0$ we find

$\Phi(\mathbf{p})=\frac{2 \Omega}{L}\left[\frac{p_{x}^{2}}{2 \pi}-\frac{1}{L} \sum_{k=-\infty}^{\infty}\left[s_{k}-\left|b_{k}+p_{x}\right|-\frac{p_{y}^{2}}{2\left|b_{k}\right|}\right]\right]$.

We have verified numerically that $\Phi(\mathbf{p})$ is positive for all p. Thus the stripe-domain structure is stable with respect to arbitrary small displacements. A statement in the literature that striped crystals are unstable under a particular infinitesimal displacement is incorrect, apparently due to an error in the evaluation of an integral. ${ }^{28}$

In order to find $K, \mu$, and $\kappa$ we must expand (A22) in a series over powers of $p_{x}$ and $p_{y}$; the coefficients of $p_{x}^{2}, p_{y}^{4}$, and $p_{x}^{2} p_{y}^{2}$ will yield the desired elastic constants. As a result, for the elastic coefficients we obtain

$$
\begin{aligned}
& K=\frac{\Omega}{\pi L}, \\
& \mu=\frac{7 \Omega L}{16 \pi^{3}} \zeta(3), \\
& \kappa=-\frac{7 \Omega L}{4 \pi^{3}} \zeta(3) .
\end{aligned}
$$

Thus, the mean-field calculation results in a negative value for the constant $\kappa$. It does not matter for the smectic crystal state, since the term proportional to $(\partial u / \partial x)^{2}$ is positive. However, this term vanishes in the Ising nematic phase, where the term $(\kappa / 2)\left(\partial^{2} u / \partial x \partial y\right)^{2}$ $=(\kappa / 2)(\partial \theta / \partial x)^{2}$ prevails. Thus the negative mean-field value of $\kappa$ can result in an instability of the Ising nematic phase. An analysis of the stabilizing effects of thermal fluctuations is given in the text and in Appendix C. 


\section{APPENDIX B: STABILITY AND ELASTIC CONSTANTS FOR $\boldsymbol{H} \neq \mathbf{0}$}

In the presence of a magnetic field we must consider two displacement fields $\left\{u_{n}\right\}$ and $\left\{v_{n}\right\}$. The equilibrium distances between nearest unperturbed domain walls are $L+\delta$ and $L-\delta$. We denote by $u_{n}$ and $v_{n}$ the displacements of the left and right domain walls bordering the $n$th stripe with the equilibrium width $L+\delta$. The Hamiltonian in this case consists of two terms the same as (A1), one for each field, an interaction term and a field term

$$
\begin{aligned}
\mathscr{H}^{(h)}= & E_{s} \sum_{n} \int\left[\sqrt{1+\left(\partial u_{n} / \partial y\right)^{2}}+\sqrt{1+\left(\partial v_{n} / \partial y\right)^{2}}\right] d y \\
& -\frac{1}{2} \sum_{m, n} \iint V\left(R_{m n}^{\prime}\right) \cos \left(\frac{\partial u_{m}}{\partial y}-\frac{\partial u_{n}}{\partial y^{\prime}}\right) \sqrt{1+\left(\partial u_{m} / \partial y\right)^{2}} \sqrt{1+\left(\partial u_{n} / \partial y^{\prime}\right)^{2}} d y d y^{\prime} \\
& -\frac{1}{2} \sum_{m, n} \iint V\left(R_{m n}^{\prime \prime}\right) \cos \left(\frac{\partial v_{m}}{\partial y}-\frac{\partial v_{n}}{\partial y^{\prime}}\right) \sqrt{1+\left(\partial v_{m} / \partial y\right)^{2}} \sqrt{1+\left(\partial v_{n} / \partial y^{\prime}\right)^{2}} d y d y^{\prime} \\
& +\sum_{m, n} \iint V\left(R_{m n}^{\prime \prime \prime}\right) \cos \left(\frac{\partial v_{m}}{\partial y}-\frac{\partial u_{n}}{\partial y^{\prime}}\right) \sqrt{1+\left(\partial v_{m} / \partial y\right)^{2}} \sqrt{1+\left(\partial u_{n} / \partial y^{\prime}\right)^{2}} d y d y^{\prime} \\
& -2 h \sum_{n} \int\left[\delta+v_{n}(y)-u_{n}(y)\right] d y,
\end{aligned}
$$

where

$R_{m n}^{\prime 2}=\left(x_{m n}+u_{m}-u_{n}\right)^{2}+\left(y-y^{\prime}\right)^{2}, \quad x_{m n}=2(m-n) L$,

$R_{m n}^{\prime \prime 2}=\left(x_{m n}+v_{m}-v_{n}\right)^{2}+\left(y-y^{\prime}\right)^{2}$,

$R_{m n}^{\prime \prime \prime 2}=\left(\widetilde{x}_{m n}+v_{m}-u_{n}\right)^{2}+\left(y-y^{\prime}\right)^{2}$,

$\widetilde{x}_{m n}=[2(m-n)+\bar{\delta}] L$.

We first obtain the equilibrium conditions, from which $L$ and $\delta$ can be found. Setting $u_{n}=0, v_{n}=0$ in (B1), and dividing by the area, we obtain

$$
E=E_{s} n-\frac{n}{2} \sum_{k} \int\left[V\left(R_{k}^{\prime}\right)-V\left(R_{k}^{\prime \prime}\right)\right] d y-h n \delta
$$

where $n=1 / L, \quad R_{k}^{\prime 2}=(2 k L)^{2}+y^{2}, \quad$ and $\quad R_{k}^{\prime \prime 2}=[(2 k$ $+\bar{\delta}) L]^{2}+y^{2}$. The integral and sum in the middle term can be rewritten by the use of (A16) and (A18) as

$$
\widehat{V}(\mathbf{p}=0)-\widehat{V}_{\sim}(\mathbf{p}=0)=\frac{\Omega}{L} \sum_{k=-\infty}^{+\infty} \frac{e^{-l\left|b_{k}\right|}-e^{i \pi k \bar{\delta}-l\left|b_{k}\right|}}{\left|b_{k}\right|}
$$

The sum gives

$$
\begin{array}{r}
\frac{2 L}{\pi} \mathcal{R}\left[\ln \left(1+e^{-l \pi / L+i \pi \delta}\right)-\ln \left(1-e^{-l \pi / L}\right)\right]_{l \ll L} \\
=\frac{2 L}{\pi} \ln \left[\frac{2}{\pi n l} \cos \frac{\pi n \delta}{2}\right],
\end{array}
$$

which then yields (48) of the text. A logarithmic term like this was obtained by Marchenko in the context of the competition between two types of facet on a crystal surface. $^{29}$

Expansion of (B1) to terms first order in $u_{n}$ and $v_{n}$, and the requirement that the state be a local minimum with respect to them, yields two conditions, equivalent to (49). Expansion (B1) to terms second order in $u_{n}$ and $v_{n}$ yields

$$
\begin{aligned}
\delta \mathscr{H}^{(h)=}= & \frac{E_{s}}{2} \sum_{n} \int\left[\left[\frac{\partial u_{n}}{\partial y}\right]^{2}+\left[\frac{\partial v_{n}}{\partial y}\right]^{2}\right] d y \\
& -\frac{1}{2} \sum_{m, n} \iint\left[V\left(R_{m n}\right)\left[\frac{\partial u_{m}}{\partial y} \frac{\partial u_{n}}{\partial y^{\prime}}+\frac{\partial v_{m}}{\partial y} \frac{\partial v_{n}}{\partial y^{\prime}}\right]-2 V\left(\widetilde{R}_{m n}\right) \frac{\partial u_{m}}{\partial y} \frac{\partial v_{n}}{\partial y^{\prime}}\right. \\
& \left.+\frac{1}{2} W\left(R_{m n}\right)\left[\left(u_{m}-u_{n}\right)^{2}+\left(v_{m}-v_{n}\right)^{2}\right]-W\left(\widetilde{R}_{m n}\right)\left(u_{m}-v_{n}\right)^{2}\right] d y d y^{\prime},
\end{aligned}
$$

where

$$
\begin{aligned}
& R_{m n}^{2}=x_{m n}^{2}+\left(y-y^{\prime}\right)^{2}, \\
& \widetilde{R}_{m n}^{2}=\widetilde{x}_{m n}^{2}+\left(y-y^{\prime}\right)^{2} .
\end{aligned}
$$

On Fourier transforming (B8) we obtain

$$
\delta \mathscr{H}^{(h)}=\frac{1}{2 L} \int\left(d^{2} p\right)\left(u_{-\mathbf{p}} v_{-\mathbf{p}}\right)\left(\begin{array}{ll}
H_{1}(\mathbf{p}) & H_{2}(\mathbf{p}) \\
H_{2}^{*}(\mathbf{p}) & H_{1}(\mathbf{p})
\end{array}\right)\left[\begin{array}{l}
u_{\mathrm{p}} \\
v_{\mathrm{p}}
\end{array}\right),
$$


where now $-\pi / 2 L \leq p_{x} \leq \pi / 2 L$ and $-\infty \leq p_{y} \leq \infty$, and

$H_{1}(\mathbf{p})=E_{s} p_{y}^{2}-\widehat{V}(\mathbf{p}) p_{y}^{2}-\hat{W}(0)+\hat{W}(\mathbf{p})+\hat{W}_{\sim}(0)$,

$H_{2}(\mathbf{p})=e^{i p_{x} L \bar{\delta}_{\delta}}\left[p_{y}^{2} \widehat{V}_{\sim}(\mathbf{p})-\widehat{W}_{\sim}(\mathbf{p})\right]$.

Thus the problem is reduced to that of a pair of coupled oscillators. Diagonalization of the matrix in (B11) yields

$\mathscr{H}_{\mathrm{el}}=\delta \mathscr{H}^{(h)}=\frac{1}{2 L} \int\left(d^{2} p\right)\left[\boldsymbol{H}_{+}\left|w_{+}\right|^{2}+H_{-}\left|w_{-}\right|^{2}\right]$,

$H_{ \pm}=H_{1} \pm\left|H_{2}\right|$,

$w_{ \pm}=\frac{1}{\sqrt{2}}\left(u \pm e^{i \phi\left(p_{x}, p_{y}, h\right)} v\right), e^{i \phi\left(p_{x}, p_{y}, h\right)}=\frac{H_{2}}{\left|H_{2}\right|}$.

$H_{+}$and $H_{-}$correspond to the optic and acoustic branches of the spectrum. Note that $|\phi(0,0,0)|=\pi$, so that $w_{+}=(u-v) / \sqrt{2}$ in zero magnetic field. Explicitly, on eliminating $E_{s}$ via Eq. (A20) (which also holds in a field, where $\bar{\delta} \neq 1$ ), we obtain

$$
\begin{aligned}
H_{ \pm}=\frac{\Omega}{L^{2}}[ & \left\{\sum_{k} e^{-l\left|a_{k}\right|}\left(1-e^{i \pi k \bar{\delta}}\right)\right. \\
& \times\left[\left|a_{k}\right|+p_{y}^{2}\left(\frac{1}{\left|a_{k}\right|}-l\right)\right] \\
& \left.\left.-p_{k} e^{-l p_{k}}\right\} \pm\left|\sum_{k} e^{i \pi k \bar{\delta}} p_{k} e^{-l p_{k}}\right|\right],
\end{aligned}
$$

where $p_{k}^{2}=\left(p_{x}+a_{k}\right)^{2}+p_{y}^{2}$ and $a_{k}=\pi k / L$.

When $p_{y}=0$ we have

$\left|H_{2}\right|=\frac{\Omega}{L^{2}}|| p_{x}\left|-\frac{\pi}{2 L} \sec ^{2} \frac{\pi n \delta}{2}-i p_{x} \tan \frac{\pi n \delta}{2}\right|$.

From (B18), it is clear that $\phi\left(p_{x} \rightarrow+0, p_{y}=0\right.$, $h \rightarrow+0)=-\pi$, as noted above. Again for $p_{y}=0$, we have

$$
\begin{array}{r}
H_{ \pm}=\frac{\Omega}{L}\left[-\left|p_{x}\right|+\frac{L}{\pi} p_{x}^{2}+\frac{\pi}{2 L} \sec ^{2}\left(\frac{\pi n \delta}{2}\right)\right. \\
\times\left\{1 \pm\left[1+\left(\frac{2 L}{\pi}\right)^{2} \cos ^{2} \frac{\pi n \delta}{2}\right.\right. \\
\left.\left.\left.\times\left[p_{x}^{2}-\frac{\pi\left|p_{x}\right|}{L}\right)\right]^{1 / 2}\right\}\right] .
\end{array}
$$

In Fig. 6, these two quantities are plotted in dimensionless units of $\omega=2 H /\left[\pi \Omega\left(n^{*}\right)^{2}\right]$ (the upper and lower curves corresponding to $H_{+}$and $H_{-}$, respectively), as a function of the dimensionless variable $p_{x} L_{0} / \pi$. Here $L_{0}$, the value of $L$ in zero field, is employed, so that the Brillouin zone moves in with increasing field.

Expansion of $H_{-}$to second order in $p_{x}^{2}$ yields the elastic constant $K$ in a magnetic field:

$$
K(h)=\frac{\Omega}{\pi L} \cos ^{2} \frac{\pi n \delta}{2} .
$$

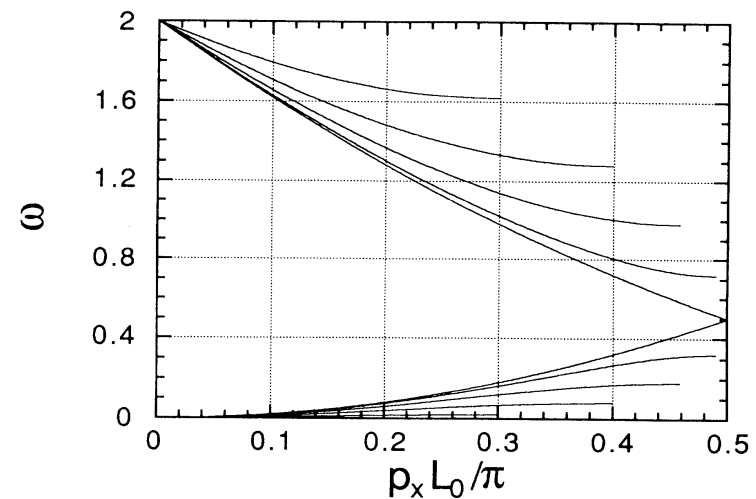

FIG. 6. Values of $H_{ \pm}$normalized as indicated in the text. Five values of the $h / h_{c}$ were chosen: $0,0.2,0.4,0.6,0.8,1.0$. For $h / h_{c}=0$, the upper and lower curves meet at the Brillouin zone; the other curves deviate from these monotonically with $h / h_{c}$. We employ $L_{0}$, the value of $L$ in zero field, so that the Brillouin zone moves toward the origin as $h / h_{c}$ increases

To find $\mu(h)$ and $\kappa(h)$ we must employ the full expression for $H_{-}$. The $p_{y}^{4}$ term yields

$$
\mu(h)=\frac{\Omega L}{4 \pi^{3}} \sum_{k=1}^{\infty} \frac{1-\cos \pi k \bar{\delta}}{k^{3}},
$$

which agrees with Eq. (A25) when $h=\delta=0$. The $p_{x}^{2} p_{y}^{2}$ term yields

$$
\kappa(h)=-\frac{\Omega L}{\pi^{3}} \sum_{k=1}^{\infty} \frac{1-\cos \pi k \bar{\delta}}{k^{3}},
$$

which agrees with Eq. (A26) for $h=\delta=0$.

\section{APPENDIX C: THERMAL RENORMALIZATION OF $\kappa$}

In Appendix B we found the elastic constant $\kappa$ to be negative. It implies an instability in the system, which would result in a first-order transition from the smectic crystal to the tetragonal liquid phase. The thermal corrections to $\kappa$ induced by the nonlinearities of the elastic Hamiltonian can stabilize the Ising nematic phase. We now determine the one-loop correction to $\kappa$.

The two-point correlation function is defined as usual:

$$
G(\mathbf{p})=\langle u(\mathbf{p}) u(-\mathbf{p})\rangle .
$$

The anharmonic terms of the Hamiltonian are

$$
\frac{K}{2}\left[\frac{\partial u}{\partial x}\left[\frac{\partial u}{\partial y}\right]^{2}+\frac{1}{4}\left[\frac{\partial u}{\partial y}\right]^{4}\right] \text {. }
$$

These two anharmonic terms occur because the bending energy actually is not $(K / 2)\left(\partial_{x} u\right)^{2}$, but $(K / 2)\left[\partial_{x} u\right.$ $\left.+\frac{1}{2}\left(\partial_{y} u\right)^{2}\right]^{2}$, a form that is invariant under rotations.

Dyson's equation is given by

$$
(G(\mathbf{p}))^{-1}=\left(G^{(0)}(\mathbf{p})\right)^{-1}-\Sigma(\mathbf{p}),
$$

with the bare correlator 


$$
G^{(0)}(\mathbf{p})=\frac{T}{K p_{x}^{2}+\mu p_{y}^{4}+v p_{y}^{2}} .
$$

All diagrams of the second order are represented in Fig. 7, where solid lines are the bare correlators, short lines intersecting solid lines represent differentiation with respect to $y$ or multiplication by $p_{y}$, short double lines represent differentiation with respect to $x$ or multiplication by $p_{x}$, a nd each solid circle is a vertex $-K / 2 T$. All internal momenta must be integrated over. Each diagram must have its combinatorial factor determined individually. To obtain the corrections to $\kappa$ we need not compute the diagrams in full detail, but only the coefficients of $p_{x}^{2} p_{y}^{2}$ in their Taylor expansions.

Two obvious relations that will be employed are

$$
\begin{aligned}
& \frac{\partial}{\partial q_{y}}\left(G^{(0)}(\mathbf{q})\right)^{n}=-\frac{2 n\left(2 \mu q_{y}^{3}+v q_{y}\right)}{T}\left(G^{(0)}(\mathbf{q})\right)^{n+1}, \\
& \frac{\partial}{\partial q_{x}}\left(G^{(0)}(\mathbf{q})\right)^{n}=-\frac{2 n K q_{x}}{T}\left(G^{(0)}(\mathbf{q})\right)^{n+1} .
\end{aligned}
$$

The graph in Fig. 7(a) gives a contribution to $\Sigma$ of

$$
2 \alpha p_{x}^{2} \int G^{(0)}(\mathbf{q}) G^{(0)}(\mathbf{p}-\mathbf{q})\left(p_{y}-q_{y}\right)^{2} q_{y}^{2}\left(d^{2} q\right),
$$

where $\alpha=(K / 2 T)^{2}$ and $\left(d^{2} q\right)=d q_{x} d q_{y} /(2 \pi)^{2}$. The coefficient of $p_{x}^{2} p_{y}^{2}$ is

$$
2 \alpha \int G\left[G+2 q_{y} \frac{\partial}{\partial q_{y}} G+\frac{q_{y}^{2}}{2} \frac{\partial^{2}}{\partial q_{y}^{2}} G\right]\left(d^{2} q\right),
$$

(a)

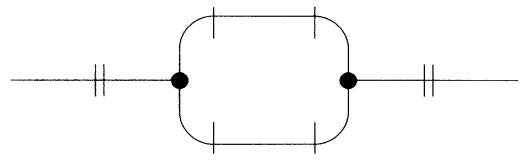

(b)

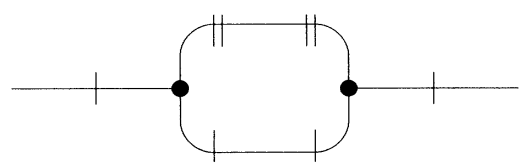

(c)

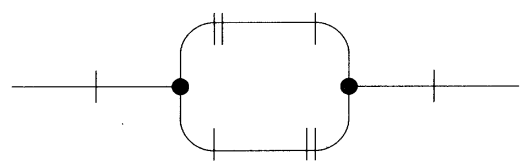

(e)

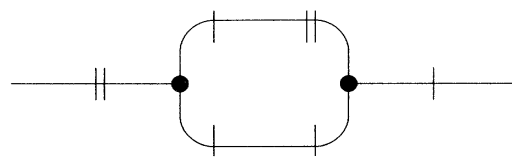

FIG. 7. Diagrams relevant to thermal renormalization of the elastic constant $\kappa$. Solid lines indicate bare correlators, with $\mathbf{p}$ entering and leaving. Single short lines indicate $y$ momentum and double short lines indicate $x$ momentum. Solid circles indicate vertices $-K / 2 T$. where we have used the obvious relation $\partial f(p-q) /\left.\partial p\right|_{p=0}=-\partial f(q) / \partial q$, and introduced $G$ instead of $G^{(0)}(q)$ for brevity. Using relation $(C 5)$ we can reduce this term to

$$
\begin{aligned}
& \alpha \int q_{y}^{2}\left[-\frac{3}{2} G^{2}+\frac{4 v q_{y}^{2}}{3 T} G^{3}\right]\left(d^{2} q\right) \\
& =\alpha T\left[\frac{3}{2} \frac{\partial}{\partial v}+\frac{2}{3} v \frac{\partial^{2}}{\partial v^{2}}\right] \int G(v)\left(d^{2} q\right)
\end{aligned}
$$

The last integral can be easily evaluated. As a result we have

$\frac{\partial T^{2}}{2 \pi \sqrt{K}}\left(\frac{3}{2} \frac{\partial}{\partial v}+\frac{2}{3} v \frac{\partial^{2}}{\partial v^{2}}\right) \frac{1}{\sqrt{v}} \sinh ^{-1} \sqrt{v / \mu} \frac{1}{p_{x}}$,

where we introduce the infrared cutoff $p_{x}$. Finally, to obtain the leading term we differentiate only $1 / \sqrt{v}$ before $\sinh ^{-1}$, thus yielding for the thermal correction to $\kappa$,

$$
\delta \kappa=-\frac{T}{64 \pi}\left[\frac{K}{v}\right]^{3 / 2} \ln \frac{p_{x}^{2} \mu}{4 v} .
$$

We can now write out the renormalized value of $\kappa$ because, as will be seen later, the other graphs give smaller contributions to $\kappa$. Thus

$$
\kappa^{\prime}=\kappa-\frac{T}{64 \pi}\left[\frac{K}{v}\right]^{3 / 2} \ln \frac{p_{x}^{2} \mu}{4 v} .
$$

We now show that the contributions from the diagrams in Figs. 7(b) and 7(c) cancel. We can consider them together because both have the same external momenta $p_{y}$. Their contribution to $\Sigma$ is

$$
\begin{aligned}
& 4 p_{y}^{2} \alpha \int G^{(0)}(\mathbf{q}) G^{(0)}(\mathbf{p}-\mathbf{q}) \\
& \quad \times\left[q_{x}^{2}\left(p_{y}-q_{y}\right)^{2}+q_{x} q_{y}\left(p_{x}-q_{x}\right)\left(p_{y}-q_{y}\right)\right]\left(d^{2} q\right) .
\end{aligned}
$$

Note that they have the same combinatorial factor. The coefficient of $p_{x}^{2} p_{y}^{2}$ is

$$
\begin{aligned}
& 4 \alpha \int q_{y}^{2} G\left[q_{x} \frac{\partial G}{\partial q_{x}}+q_{x}^{2} \frac{\partial^{2} G}{\partial q_{x}^{2}}\right]\left(d^{2} q\right) \\
& =4 \alpha\left[-\frac{2 K}{T}\right] \int 2 q_{y}^{2} q_{x}^{2}\left[G^{3}+\frac{1}{3} q_{x} \frac{\partial}{\partial q_{x}} G^{3}\right]\left(d^{2} q\right)
\end{aligned}
$$

where we have used (C6). This integral, on integration by parts, is zero.

The contribution from the diagram in Fig. 7(d) to $\Sigma$ is

$8 \alpha p_{x} p_{y} \int G^{(0)}(\mathbf{q}) G^{(0)}(\mathbf{p}-\mathbf{q}) q_{x} q_{y}\left(p_{y}-q_{y}\right)^{2}\left(d^{2} q\right)$.

The coefficient of $p_{x}^{2} p_{y}^{2}$ is

$$
8 \alpha \int q_{x} q_{y} G\left[q_{y}^{2} \frac{\partial^{2}}{\partial q_{x} \partial q_{y}} G+2 q_{y} \frac{\partial}{\partial q_{x}} G\right]\left(d^{2} q\right) .
$$

On application of (C5) and (C6) this becomes 
$8 \alpha \frac{2 K}{T} \int\left(q_{x} q_{y}\right)^{2}\left(-\frac{2 q_{y}}{3} \frac{\partial}{\partial q_{y}} G^{3}-2 G^{3}\right)\left(d^{2} q\right)$,

which gives zero, on integration by parts.

So far we have considered the one-loop contributions to $\kappa$, which yielded a term logarithmic in the external momentum. We now show that many-loop diagrams do not yield logarithmic terms, but rather terms that vary as a power of the external momentum.

Each line connecting two vertices has zero momentum dimension because it includes both the bare correlator, which is proportional to $p^{-2}$ (its $p^{4}$ term is irrelevant since the divergence occurs when $p=0$ ), and the factors of $p$, one for each vertex. Each loop implies an integration over internal momentum, i.e., gives an extra $p^{2}$. Because the momentum dependence of the interaction has been transferred to the lines, the vertices contribute factors proportional to $(K / T)$. Finally, to find a correction to $\kappa$ one must differentiate a diagram with respect to momentum twice. The result of counting the powers of momentum is $2 L-2$, where $L$ is a number of the loops in a diagram. Clearly, only the one-loop correction $(L=1)$ can be logarithmic. For example, the contribution of the diagram in Fig. 7(a) is of second order in $p$.

Let us now perform a more quantitative analysis. We consider a diagram with $V=V_{3}+V_{4}$ vertices (where $V_{3}$ and $V_{4}$ are the numbers of vertices with 3 and 4 legs, respectively), $L$ loops, and $N$ lines. These four quantities are not independent. They satisfy the two relations

$$
\begin{aligned}
& N=\frac{1}{2}\left(3 V_{3}+4 V_{4}-2\right), \\
& L=\frac{1}{2} V_{3}+V_{4} .
\end{aligned}
$$

In order to evaluate any diagram one must find ultraviolet cutoffs $\bar{p}_{x}$ and $\bar{p}_{y}$. They can be found by comparison of the terms of second and fourth orders in $p$ in the bare correlator. The result is

$$
\bar{p}_{x}=\frac{v}{\sqrt{K \mu}}, \quad \bar{p}_{y}=\sqrt{v / \mu}
$$

Using the fact that a $V_{3}$ vertex has one leg multiplied by $p_{x}$ and two legs multiplied by $p_{y}$, and a $V_{4}$ vertex has four legs multiplied by $p_{y}$, for any diagram one obtains schematically

$$
\left\lfloor\frac{K}{T}\right\rfloor^{V} \int \frac{T^{N} p_{x}^{n} p_{y}^{m}}{\left(K p_{x}^{2}+v p_{y}^{2}\right)^{N}}\left(d^{2} p\right)^{L},
$$

where $n=V_{3}-2$ and $m=2 V_{3}+4 V_{4}-2$. Rescaling the momenta via $q_{x}=\sqrt{K} p_{x}$ and $q_{y}=\sqrt{v} p_{y}$, from $(\mathrm{C} 21)$ one obtains, to within a numerical factor, the expression

$T^{N-V_{K}} K^{V-\left(V_{3}-2+L\right) / 2} v^{-\left(2 V_{3}+4 V_{4}-2+L\right) / 2} \bar{q}^{2 L-2}$,

where $\bar{q}=v / \sqrt{\mu}$ is the common ultraviolet cutoff for $q_{x}$ and $q_{y}$. This agrees with the result obtained above from power counting. Using (C18) and (C19), the correction to $\kappa$ goes as

$$
T\left(\frac{K}{v}\right)^{3 / 2}\left[\frac{T}{\mu}\left[\frac{K}{v}\right)^{1 / 2}\right]^{L-1} .
$$

For $L=1$ we obtain the previous result [Eq. (C11)],

$$
T\left(\frac{K}{v}\right)^{3 / 2} \ln \frac{p_{x}^{2} \mu}{v},
$$

where the logarithm occurs because $q$ in Eq. (C22) has an exponent of zero, so we must employ the infrared cutoff $p_{x}$. For $T \approx T_{P} \sim \sqrt{K v} L^{2}$, the factor in square brackets in (C23) becomes a numerical constant on the order of unity. This estimate remains invariant under renormalization. Thus all multiple graphs contribute to $\kappa$ values of the same order of magnitude, differing only by a numerical factor, whereas the one-loop contributions contain a large logarithmic factor.
${ }^{1}$ A. B. Kashuba and V. L. Pokrovsky, Phys. Rev. Lett. 70, 3155 (1993).

${ }^{2}$ A. B. Kashuba and V. L. Pokrovsky, Phys. Rev. B 48, 10335 (1993).

3J. J. Krebs, B. T. Jonker, and G. A. Prinz, J. Appl. Phys. 63, 3467 (1988).

${ }^{4}$ M. Stampanoni et al., Phys. Rev. Lett. 59, 2483 (1987).

${ }^{5}$ D. Pescia et al., Phys. Rev. Lett. 58, 2126 (1987).

${ }^{6}$ D. P. Pappas, K. P. Kaemper, and H. Hopster, Phys. Rev. Lett. 64, 3179 (1990).

${ }^{7}$ R. Allenspach and A. Bischof, Phys. Rev. Lett. 69, 3385 (1992).

${ }^{8}$ R. Allenspach, M. Stampanoni, and A. Bischof, Phys. Rev. Lett. 65, 3344 (1990).

${ }^{9}$ Z. Q. Qiu, J. Pearson, and S. D. Bader, Phys. Rev. Lett. 70, 1006 (1993).

${ }^{10}$ L. Néel, J. Phys. Radiat. 15, 376 (1954).

${ }^{11}$ J. G. Gay and R. Richter, Phys. Rev. Lett. 56, 2728 (1986).

${ }^{12}$ V. L. Berezinskii, Zh. Eksp. Teor. Fiz. 59, 907 (1970) [Sov. Phys. JETP 32, 493 (1971)]; 61, 1144 (1971) [34, 610 (1971)].

${ }^{13}$ J. M. Kosterlitz and D. J. Thouless, J. Phys. C 6, 1181 (1973); J. M. Kosterlitz, ibid. 7, 1046 (1974); see A. P. Young, Phys. Rev. B 19, 1855 (1979), for a particularly concise discussion.
${ }^{14}$ D. Pescia and V. L. Pokrovsky, Phys. Rev. Lett. 65, 2599 (1990).

${ }^{15}$ A. P. Levanyuk and N. Garcia, Phys. Rev. Lett. 70, 1184 (1993).

${ }^{16}$ D. Pescia and V. L. Pokrovsky, Phys. Rev. Lett. 70, 1185 (1993).

${ }^{17}$ L. D. Landau and E. M. Lifshitz, Electrodynamics of Continuous Media, 2nd ed. (Pergamon, London, 1984), p. 147.

${ }^{18}$ N. D. Mermin, Phys. Rev. B 176, 250 (1968).

${ }^{19}$ B. Jancovici, Phys. Rev. Lett. 19, 20 (1967).

${ }^{20}$ G. Grinstein and R. A. Pelcovits, Phys. Rev. A 26, 915 (1982).

${ }^{21}$ J. Toner and D. R. Nelson, Phys. Rev. B 23, 316 (1981).

${ }^{22}$ I. Lyuksyutov, A. G. Naumovets, and V. Pokrovsky, TwoDimensional Crystals (Academic, San Diego, CA, 1992).

${ }^{23}$ Note that there were misprints for $\mu$ in Refs. 1 and 2. The correct value for $\mu$, as derived in Appendix A, is given by $\mu=7 \Omega L \zeta(3) / 16 \pi^{3}$.

${ }^{24}$ The last relation is given in Refs. 1 and 2, but it is easily deduced by employing those works and the following considerations. On scaling via $u \rightarrow Z_{u} u, x \rightarrow Z_{x} x, y \rightarrow Z_{y} y$, the coefficients in the elastic energy (integrated over space) have the following renormalization factors: $K$ by $Z_{u}^{2} Z_{x}^{-1} Z_{y}=Z^{2}$, 
$\mu$ by $Z_{u}^{2} Z_{x} Z_{y}^{-3}=Z^{-1}, v$ by $Z_{u}^{2} Z_{x} Z_{y}^{-1}=Z^{-1}$, and $\kappa$ by $Z_{u}^{2} Z_{x}^{-1} Z_{y}^{-1}$. Comparison of the terms for $\mu$ and $v$ yields $Z_{y}=1$, from which it follows that $\kappa$ gets renormalized in the same way as $K$.

${ }^{25}$ B. I. Halperin and D. R. Nelson, Phys. Rev. Lett. 41, 121 (1978); D. R. Nelson and B. I. Halperin, Phys. Rev. B 19, 2456 (1979).
${ }^{26}$ P. G. DeGennes, The Physics of Liquid Crystals (Clarendon, Oxford, 1974).

${ }^{27}$ Y. Yafet and E. M. Gyorgy, Phys. Rev. B 38, 9145 (1988).

${ }^{28}$ V. I. Marchenko, Zh. Eksp. Teor. Fiz. 90, 2241 (1986) [Sov. Phys. JETP 63, 1315 (1986)].

${ }^{29}$ V. I. Marchenko, Zh. Eksp. Teor. Fiz. 81, 1141 (1981) [Sov. Phys. JETP 54, 605 (1981)]. 